\title{
Nonlinear Beamforming Based on Group-Sparsities of Periodograms for Phased Array Weather Radar
}

\author{
Daichi Kitahara, Member, IEEE, Hiroki Kuroda, Member, IEEE, Akira Hirabayashi, Member, IEEE, \\ Eiichi Yoshikawa, Hiroshi Kikuchi, and Tomoo Ushio, Member, IEEE
}

\begin{abstract}
We propose nonlinear beamforming for phased array weather radars (PAWRs). Conventional beamforming is linear in the sense that a backscattered signal arriving from each elevation is reconstructed by a weighted sum of received signals, which can be seen as a linear transform for the received signals. For distributed targets such as raindrops, however, the number of scatterers is significantly large, differently from the case of point targets that are standard targets in array signal processing. Thus, the spatial resolution of the conventional linear beamforming is limited. To improve the spatial resolution, we exploit two characteristics of a periodogram of each backscattered signal from the distributed targets. The periodogram is a series of the powers of the discrete Fourier transform (DFT) coefficients of each backscattered signal and utilized as a nonparametric estimate of the power spectral density. Since each power spectral density is proportional to the Doppler frequency distribution, (i) major components of the periodogram are concentrated in the vicinity of the mean Doppler frequency, and (ii) frequency indices of the major components are similar between adjacent elevations. These are expressed as group-sparsities of the DFT coefficient matrix of the backscattered signals, and we propose to reconstruct the signals through convex optimization exploiting the group-sparsities. We consider two optimization problems which evaluate the groupsparsities in slightly different ways, and both problems are solved with the alternating direction method of multipliers including nonlinear mappings. Simulations using real PAWR data show that the proposed method dramatically improves the spatial resolution.
\end{abstract}

Index Terms-Phased array weather radar, distributed target, beamforming, periodogram, group-sparsity, convex optimization.

\section{INTRODUCTION}

$\mathbf{P}$ HASED array weather radars (PAWRs) [1]-[6] have been developed to quickly detect hazardous weather phenomena such as a thunderstorm with heavy rain. Classical parabolic

Manuscript received $\mathrm{xxxxxx} \mathrm{xx}, 2021$; revised $\mathrm{xxxxxx} \mathrm{xx}$ and $\mathrm{xxxxxx} \mathrm{xx}$; accepted $\operatorname{xxxxxx} x x, 202 x$. Date of publication $\operatorname{xxxxxx} x x, 202 x$; date of current version $\operatorname{xxxxxx} \mathrm{xx}, 202 \mathrm{x}$. This work was supported in part by JSPS Grants-in-Aid (JP19K20361). (Corresponding author: Daichi Kitahara.)

D. Kitahara, H. Kuroda, and A. Hirabayashi are with the College of Information Science and Engineering, Ritsumeikan University, Shiga 525-8577, Japan (e-mail: \{d-kita; kuroda; akirahrb\}@media.ritsumei.ac.jp).

E. Yoshikawa is with the Aeronautical Technology Directorate, Japan Aerospace Exploration Agency, Tokyo 181-0015, Japan (e-mail: yoshikawa. eiichi@jaxa.jp).

H. Kikuchi is with the Center for Space Science and Radio Engineering, The University of Electro-Communications, Tokyo 182-8585, Japan (e-mail: hkikuchi@uec.ac.jp).

T. Ushio is with the Division of Electrical, Electronic and Information Engineering, Osaka University, Osaka 565-0871, Japan (e-mail: ushio@eei.eng. osaka-u.ac.jp).

Color versions of one or more of the figures in this article are available online at http://ieeexplore.ieee.org.

Digital Object Identifier 10.1109/TGRS.2021.xxxxxxx
Doppler weather radars [7], [8] transmit a pencil beam and receive backscattered signals within a narrow range of elevation angles. By repeatedly transmitting and receiving the signals at several elevation angles with the mechanical vertical scan, the classical Doppler radar can observe the weather in the sky. On the other hand, PAWRs transmit a fan beam and receive backscattered signals within a wide range of elevation angles by an array antenna. Then, the backscattered signals within the narrow ranges are reconstructed from the received signals of the array antenna by digital beamforming [9]-[20]. This is the key technology in the PAWR because it gets rid of the mechanical vertical scan and hence the temporal resolution is significantly improved in the weather observation. For example, the PAWR developed at Osaka University [5] can observe the weather of a radius 60 kilometers in 30 seconds, while the classical Doppler radar requires 5 to 10 minutes for a similar observation [8].

Major beamforming methods [9]-[19] reconstruct the signal arriving from each elevation as a complex weighted sum of the received signals. In particular, Capon beamforming [12]-[17], also known as the minimum variance distortionless response (MVDR) beamforming [18], is widely used since it can adaptively reduce the influence of so-called sidelobes if a sufficient number of beams (pulses) are transmitted. However, for quick weather observations, the number of pulses should be as small as possible. To deal with such a situation, the minimum mean square error (MMSE) beamforming [19] was proposed. In this method, differently from Capon's method, the covariance matrix of the received signals is iteratively updated, and hence the backscattered signal arriving from each elevation can be reconstructed robustly even if the number of the pulses is small.

Beamforming methods were developed originally for observations of point targets in array signal processing, while targets of the PAWR are distributed targets such as raindrops. In this case, the number of scatterers is significantly large, i.e., much greater than the number of antenna elements. As a result, the spatial resolution of the above linear methods [9]-[19] is limited, and fine fluctuations of the average signal power densities corresponding to precipitation profile have not been captured.

To overcome the limitation of the linear methodology, this paper proposes a nonlinear beamforming method. We formulate the beamforming as an ill-conditioned inverse problem. To solve it, we exploit properties of periodograms of the backscattered signals from the distributed targets. The periodogram is a series of the powers of the discrete Fourier transform (DFT) coefficients of each backscattered signal and often used as a nonparametric estimator for the power spectral density of the 
backscattered signal. Since the power spectral density is proportional to the Doppler frequency distribution [7], (i) dominant components of each periodogram are concentrated in the vicinity of the mean Doppler frequency, and (ii) frequency indices of the dominant components are similar between the periodograms of adjacent elevation angles. We express these two properties as group-sparsities of the DFT coefficient matrix of the backscattered signals and propose to reconstruct the DFT coefficient matrix by minimizing a cost function that consists of a data-fidelity term and two group $\ell_{1}$-norms evaluating the group-sparsities. We consider two optimization problems that compute the group $\ell_{1}$-norms in different ways, and both problems are solved by the alternating direction method of multipliers (ADMM) [21]-[24]. Since nonlinear mappings called the proximity operators are used in the ADMM iterations, the proposed beamforming is a nonlinear transform for the received signals. Numerical simulations based on real PAWR data show that the proposed nonlinear beamforming greatly improves the spatial resolution in comparison with the linear beamforming.

The rest of this paper is organized as follows. In Section II, after explaining mathematical notation, we introduce the basic principles of the classical Doppler weather radar, and then we formulate the observation model in the PAWR. In Section III, we describe three conventional linear beamforming [11], [12], [19]. Section IV presents the proposed nonlinear beamforming. First, we derive the data-fidelity term in the frequency domain. Second, we explain the group $\ell_{1}$-norms evaluating two groupsparsities of periodograms in Section IV-A and Section IV-B. Third, we propose two convex optimization problems including their ADMM iterations in Section IV-C and Section IV-D. Section $\mathrm{V}$ shows the effectivity of the proposed beamforming by simulations, and finally Section VI concludes this paper.

\section{Preliminaries}

Let $\mathbb{R}, \mathbb{R}_{+}$, and $\mathbb{C}$ be the sets of all real numbers, nonnegative real numbers, and complex numbers, respectively. We use $j \in \mathbb{C}$ to denote the imaginary unit, i.e., $j:=\sqrt{-1}$. For any complex number $x \in \mathbb{C}, \bar{x}$ denotes its complex conjugate, and $|x|:=\sqrt{x \bar{x}}$ denotes its absolute value. We write vectors and matrices by boldface small and capital letters, respectively. We use $\boldsymbol{I}_{n} \in\{0,1\}^{n \times n}$ to denote the identity matrix of order $n$. The transpose and Hermitian transpose of a vector or a matrix are denoted by $(\cdot)^{\mathrm{T}}$ and $(\cdot)^{\mathrm{H}}$, respectively. The $\ell_{2}$-norm (or the Euclidean norm) of a vector $\boldsymbol{x}:=\left(x_{1}, x_{2}, \ldots, x_{n}\right)^{\mathrm{T}} \in \mathbb{C}^{n}$ is defined by $\|\boldsymbol{x}\|_{2}:=\sqrt{\sum_{i=1}^{n}\left|x_{i}\right|^{2}}$. A weighted group $\ell_{1}$-norm ${ }^{1}$ of $\boldsymbol{x}$ is defined, with a non-overlapping partition $G:=\left(\mathcal{G}_{i}\right)_{i=1}^{n_{G}}$ and a nonnegative vector $\boldsymbol{\xi}:=\left(\xi_{1}, \xi_{2}, \ldots, \xi_{n_{G}}\right)^{\mathrm{T}} \in \mathbb{R}_{+}^{n_{G}}$, by $\|\boldsymbol{x}\|_{1, \boldsymbol{\xi}}^{G}:=\sum_{i=1}^{n_{G}} \xi_{i}\left\|\boldsymbol{x}_{\mathcal{G}_{i}}\right\|_{2}$, where $\mathcal{G}_{i}(\neq \varnothing)$ is an index set for the $i$ th group s.t. $\bigcup_{i=1}^{n_{G}} \mathcal{G}_{i}=\{1,2, \ldots, n\}$ and $\mathcal{G}_{i_{1}} \cap \mathcal{G}_{i_{2}}=\varnothing$ $\left(i_{1} \neq i_{2}\right)$, and $\boldsymbol{x}_{\mathcal{G}}:=\left(x_{i}\right)_{i \in \mathcal{G}}$ is the subvector ${ }^{2}$ extracted from $\boldsymbol{x}$ with $\mathcal{G}$. The Frobenius norm of a matrix $\boldsymbol{X}:=\left(x_{i_{1}, i_{2}}\right) \in$ $\mathbb{C}^{n_{1} \times n_{2}}$ is defined by $\|\boldsymbol{X}\|_{\mathrm{F}}:=\sqrt{\sum_{i_{1}=1}^{n_{1}} \sum_{i_{2}=1}^{n_{2}}\left|x_{i_{1}, i_{2}}\right|^{2}}$. We use $E[\cdot]$ to denote the expected value of some random variable.

\footnotetext{
${ }^{1}$ We also define a weighted group $\ell_{1}$-norm of a matrix $\boldsymbol{X}$ in a similar way.

${ }^{2}$ If $\boldsymbol{x}_{\mathcal{G}_{i}} \approx \mathbf{0}$ for many groups $\mathcal{G}_{i}, \boldsymbol{x}$ is called group-sparse. In particular, if all $\mathcal{G}_{i}$ consist only of consecutive indices, $\boldsymbol{x}$ is also called block-sparse [25].
}

\section{A. Basic Principles of Weather Observation by Doppler Radar}

Classical parabolic Doppler weather radars transmit pulses for a specific elevation angle and receive backscattered signals generated by the incidence of the transmitted signals to targets (clouds and raindrops). For a fixed range $r[\mathrm{~m}]$, the $l$ th discretetime sample $\widehat{x}_{l} \in \mathbb{C}$ of the received signal is expressed as

$\widehat{x}_{l}:=x_{l}+\varepsilon_{l}:=x\left((l-1) T+\frac{2 r}{c}\right)+\varepsilon_{l} \quad(l=1,2, \ldots, L)$,

where $c[\mathrm{~m} / \mathrm{s}]$ is the speed of light, $T[\mathrm{~s}]$ is the pulse repetition time satisfying $T \geq \frac{2 r}{c}, L$ is the number of the pulses, $x_{l} \in \mathbb{C}$ is the sample of the backscattered signal $x(t)$ generated by the pulse transmitted at $t=(l-1) T$, and $\varepsilon_{l} \in \mathbb{C}$ is additive white Gaussian noise of variance $E\left[\left|\varepsilon_{l}\right|^{2}\right]=\sigma_{\varepsilon}^{2}$. We consider $x(t)$ to be one sample realization of a continuous-time random process $X(t)$ which is wide-sense stationary, ${ }^{3}$ ergodic ${ }^{4}$ and zero-mean, i.e., $\mu_{x}:=E[X(t)]=0$, and whose autocorrelation function $R_{x}(\tau):=E[X(t+\tau) \overline{X(t)}]$ satisfies $\int_{-\infty}^{\infty}\left|\tau R_{x}(\tau)\right| \mathrm{d} \tau<\infty$.

Strictly speaking, $x(t)$ is expressed as the sum of individual backscattered signals in the scattering resolution volume [7]:

$$
x(t)=\sum_{s} a_{s}(t) e^{j\left(2 \pi f_{s} t+\phi_{s}\right)},
$$

where $a_{s}(t)>0$ is the scattering amplitude of the $s$ th particle, $f_{s}[\mathrm{~Hz}]$ is called the Doppler frequency, which is independently generated from a common probability density function $q(f)$, and $\phi_{s}[\mathrm{rad}]$ is called the phase shift, which is uniformly distributed from $[-\pi, \pi)$ under the assumption that precipitation profile is uniform within the scattering resolution volume. The power spectral density of the backscattered signal $x(t)$ is given by the Fourier transform of the autocorrelation function $R_{x}(\tau)$ :

$$
S(f):=\int_{-\infty}^{\infty} R_{x}(\tau) e^{-j 2 \pi f \tau} \mathrm{d} \tau .
$$

Suppose that the amount of rainfall is constant and the shapes of all raindrops are the same, i.e., $\forall s \forall t a_{s}(t)=a$, during the observation. As a result, we have $R_{x}(\tau)=E\left[a^{2} \sum_{s} e^{j 2 \pi f_{s} \tau}\right]$, and the power spectral density $S(f)$ in (2) can be expressed as

$$
S(f)=E\left[a^{2} \sum_{s} \delta\left(f-f_{s}\right)\right]=P q(f)=\frac{\lambda P}{2} q_{v}\left(\frac{\lambda f}{2}\right) .
$$

In (3), $\delta(f)$ is the delta function, $P:=R_{x}(0)=E\left[\left|x_{l}\right|^{2}\right]$ is the average signal power density, $\lambda[\mathrm{m}]$ is the carrier wavelength, and $q_{v}: \mathbb{R} \rightarrow \mathbb{R}_{+}$is a common probability density function for each Doppler velocity $v_{s}=\frac{\lambda f_{s}}{2}[\mathrm{~m} / \mathrm{s}]$, where the direction of approach to the radar is defined as the positive direction of the Doppler velocity. From (3), we can see that the power spectral

\footnotetext{
${ }^{3}$ Let $X(t)$ be a continuous-time random process. $X(t)$ is called wide-sense stationary or weak-sense stationary if its mean and autocorrelation function do not vary by shifts in the time $t$, i.e., $\forall t E[X(t)]=\int_{\mathbb{C}} x q(x \mid t) \mathrm{d} x=\mu_{x}$ and $\forall t \forall \tau E[X(t+\tau) \overline{X(t)}]=\iint_{\mathbb{C}^{2}} x_{1} \bar{x}_{2} q\left(x_{1}, x_{2} \mid t+\tau, t\right) \mathrm{d} x_{1} \mathrm{~d} x_{2}=R_{x}(\tau)$, where $q(x \mid t)$ is the probability density function of $x(t)$ and $q\left(x_{1}, x_{2} \mid t+\tau, t\right)$ is the joint probability density function of $x_{1}:=x(t+\tau)$ and $x_{2}:=x(t)$.

${ }^{4}$ A wide-sense stationary process $X(t)$ is called ergodic if any sample realization $x(t)$ of the process $X(t)$ satisfies $\lim _{T \rightarrow \infty} \frac{1}{T} \int_{0}^{T} x(t) \mathrm{d} t=\mu_{x}$ and $\lim _{T \rightarrow \infty} \frac{1}{T} \int_{0}^{T} x(t+\tau) \overline{x(t)} \mathrm{d} t=R_{x}(\tau)$ by the mean-square convergence.
} 
density $S(f)$ is proportional to the Doppler frequency distribution $q(f)$ [7]. On the Doppler frequency, we call its mean

$$
\mu_{f}:=\int_{-\infty}^{\infty} f q(f) \mathrm{d} f=\int_{-\infty}^{\infty} f \frac{S(f)}{P} \mathrm{~d} f
$$

the mean Doppler frequency, and we call its standard deviation

$$
\sigma_{f}:=\sqrt{\int_{-\infty}^{\infty}\left(f-\mu_{f}\right)^{2} q(f) \mathrm{d} f}=\sqrt{\int_{-\infty}^{\infty}\left(f-\mu_{f}\right)^{2} \frac{S(f)}{P} \mathrm{~d} f}
$$

the Doppler frequency width. Similarly, we call $\mu_{v}=\frac{\lambda \mu_{f}}{2}$ the mean Doppler velocity and call $\sigma_{v}=\frac{\lambda \sigma_{f}}{2}$ the Doppler velocity width. The main purpose of the Doppler weather radars is to estimate $P, \mu_{f}$ (or $\mu_{v}$ ) and $\sigma_{f}$ (or $\sigma_{v}$ ) from the measurements $\widehat{x}_{l}$ in (1), since these parameters correspond to the amount of rainfall, the mean wind speed and the variation of wind speeds.

As shown in (3), $S(f)$ has information about $P$ and $q(f)$. However, the backscattered signal $x(t)$ is observed in discretetime, and we have to consider the power spectral density of $x_{l}$ that is given by the discrete-time Fourier transform of $R_{x}(l T)$ :

$$
S^{\langle\mathrm{d}\rangle}(f):=\sum_{l=-\infty}^{\infty} R_{x}(l T) e^{-j 2 \pi f l T}=\frac{1}{T} \sum_{n=-\infty}^{\infty} S\left(f-\frac{n}{T}\right) .
$$

If $S(f)=0$ for $|f| \geq \frac{1}{2 T}, S(f)$ can be perfectly reconstructed from $S^{\langle\mathrm{d}\rangle}(f)$. To estimate $S^{\langle\mathrm{d}\rangle}(f)$ in (4) with finite samples $x_{l}$ $(l=1,2, \ldots, L)$, we suppose that the number $L$ is even, and we compute the normalized DFT coefficients $u_{k} \in \mathbb{C}$ of $x_{l}$ by

$$
u_{k}:=\frac{1}{\sqrt{L}} \sum_{l=1}^{L} x_{l} e^{-j \frac{2 \pi(k-1-L / 2)(l-1)}{L}} \quad(k=1,2, \ldots, L) .
$$

Then, we compute a periodogram as a series of the powers of the normalized DFT coefficients $u_{k}$ :

$$
\left|u_{k}\right|^{2}=\frac{1}{L}\left|\sum_{l=1}^{L} x_{l} e^{-j \frac{2 \pi(k-1-L / 2)(l-1)}{L}}\right|^{2} \quad(k=1,2, \ldots, L) .
$$

The expected value of the periodogram $\left(\left|u_{k}\right|^{2}\right)_{k=1}^{L}$ is given by

$$
\begin{aligned}
E\left[\left|u_{k}\right|^{2}\right] & =\frac{1}{L} E\left[\sum_{l_{1}=1}^{L} \sum_{l_{2}=1}^{L} x_{l_{1}} \bar{x}_{l_{2}} e^{-j \frac{2 \pi(k-1-L / 2)\left(l_{1}-l_{2}\right)}{L}}\right] \\
& =\frac{1}{L} \sum_{l_{1}=1}^{L} \sum_{l_{2}=1}^{L} E\left[x_{l_{1}} \bar{x}_{l_{2}}\right] e^{-j \frac{2 \pi(k-1-L / 2)\left(l_{1}-l_{2}\right)}{L}} \\
& =\frac{1}{L} \sum_{l_{1}=1}^{L} \sum_{l_{2}=1}^{L} R_{x}\left(\left(l_{1}-l_{2}\right) T\right) e^{-j \frac{2 \pi(k-1-L / 2)\left(l_{1}-l_{2}\right)}{L}} \\
& =\sum_{l=-(L-1)}^{L-1}\left(1-\frac{|l|}{L}\right) R_{x}(l T) e^{-j \frac{2 \pi(k-1-L / 2) l}{L}}
\end{aligned}
$$

From (4), (7), and $\sum_{l=-\infty}^{\infty}\left|l R_{x}(l T)\right|<\infty$, we have

$$
\lim _{L \rightarrow \infty} E\left[\left|u_{k}\right|^{2}\right]=S^{\langle\mathrm{d}\rangle}\left(\frac{k-1-L / 2}{L T}\right) .
$$

Therefore, the periodogram is often used as a nonparametric estimator for $S^{\langle\mathrm{d}\rangle}(f)$. Moreover, the parameters $P, \mu_{f}$ and $\sigma_{f}$ can be estimated from the periodogram $\left(\left|u_{k}\right|^{2}\right)_{k=1}^{L}$, e.g., by

$$
\left.\begin{array}{rl}
\widehat{P} & =\frac{1}{L} \sum_{l=1}^{L}\left|x_{l}\right|^{2}=\frac{1}{L} \sum_{k=1}^{L}\left|u_{k}\right|^{2} \\
\widehat{\mu}_{f} & =\frac{1}{\widehat{P} L} \sum_{k=1}^{L} \frac{k-1-L / 2}{L T}\left|u_{k}\right|^{2} \\
\widehat{\sigma}_{f} & =\sqrt{\frac{1}{\widehat{P} L} \sum_{k=1}^{L}\left(\frac{k-1-L / 2}{L T}-\widehat{\mu}_{f}\right)^{2}\left|u_{k}\right|^{2}}
\end{array}\right\} .
$$

\section{B. Observation Model in Phased Array Weather Radar}

First of all, we show the observation model for $K$ point targets. Let a PAWR have an $N$-element uniform linear array with the inter-element spacing $d[\mathrm{~m}]$. A plane wave signal scattered from the $\kappa$ th point target hits on the array antenna at an unknown angle $\theta_{\kappa}^{\star} \in\left[-\frac{\pi}{2}, \frac{\pi}{2}\right]$, where $\theta_{1}^{\star}<\theta_{2}^{\star}<\cdots<\theta_{K}^{\star}[\mathrm{rad}]$. The $l$ th time sample $\boldsymbol{y}_{l} \in \mathbb{C}^{N}$ of the received signal is given by

$$
\boldsymbol{y}_{l}=\sum_{\kappa=1}^{K} x_{\kappa, l}^{\star} \boldsymbol{a}\left(\theta_{\kappa}^{\star}\right)+\boldsymbol{\varepsilon}_{l} \quad(l=1,2, \ldots, L),
$$

where $x_{\kappa, l}^{\star} \in \mathbb{C}$ is the $l$ th time sample of the $\kappa$ th plane wave signal, $\boldsymbol{a}\left(\theta_{\kappa}^{\star}\right) \in \mathbb{C}^{N}$ is the so-called steering vector defined by

$$
\boldsymbol{a}(\theta):=\left(1, e^{-j \frac{2 \pi d \sin \theta}{\lambda}}, e^{-j \frac{4 \pi d \sin \theta}{\lambda}}, \ldots, e^{-j \frac{2(N-1) \pi d \sin \theta}{\lambda}}\right)^{\mathrm{T}},
$$

and $\varepsilon_{l} \in \mathbb{C}^{N}$ is additive white Gaussian noise, independent of the signals $x_{\kappa, l}^{\star}$, of covariance matrix $\boldsymbol{R}_{\varepsilon}:=E\left[\varepsilon_{l} \varepsilon_{l}^{\mathrm{H}}\right]=\sigma_{\varepsilon}^{2} \boldsymbol{I}_{N}$.

On the other hand, our targets such as raindrops are called distributed targets [19], that are supposed to exist continuously (strictly speaking, a sufficient number of raindrops exist within the antenna beamwidth). In this case, the number $K$ of scatterers (raindrops) is too large, and hence the observation model in (9) is no longer appropriate. We observe the distributed targets by dividing the whole angular interval $\left[\theta_{\min }, \theta_{\max }\right] \subset\left[-\frac{\pi}{2}, \frac{\pi}{2}\right]$ into $M$ subintervals $\left[\theta_{m}-\frac{\Delta \theta}{2}, \theta_{m}+\frac{\Delta \theta}{2}\right](m=1,2, \ldots, M)$, where $\Delta \theta:=\frac{\theta_{\max }-\theta_{\min }}{M}$ and $\theta_{m}:=\theta_{\min }+\left(m-\frac{1}{2}\right) \Delta \theta$. Thus, instead of (9), we use the following observation model

$$
\boldsymbol{y}_{l}=\sum_{m=1}^{M} x_{m, l} \boldsymbol{a}_{m}+\boldsymbol{\varepsilon}_{l}=\boldsymbol{A} \boldsymbol{x}_{l}+\boldsymbol{\varepsilon}_{l} \quad(l=1,2, \ldots, L),
$$

where $x_{m, l} \in \mathbb{C}$ is the $l$ th sample of the sum of backscattered plane wave signals in the $m$ th subinterval $\left[\theta_{m}-\frac{\Delta \theta}{2}, \theta_{m}+\frac{\Delta \theta}{2}\right]$, $\boldsymbol{x}_{l}:=\left(x_{1, l}, x_{2, l}, \ldots, x_{M, l}\right)^{\mathrm{T}} \in \mathbb{C}^{M}, \boldsymbol{a}_{m}:=\boldsymbol{a}\left(\theta_{m}\right) \in \mathbb{C}^{N}$, and $\boldsymbol{A}:=\left[\begin{array}{llll}\boldsymbol{a}_{1} & \boldsymbol{a}_{2} & \cdots & \boldsymbol{a}_{M}\end{array}\right] \in \mathbb{C}^{N \times M}$. Note that the signal $x_{l}$ in (1) corresponds to $x_{m, l}$ in (10) for a specific elevation angle $\theta_{m}$. In the PAWR system, $\theta_{m}$ is the $m$ th elevation angle, and the average signal power density vector

$$
\begin{aligned}
\boldsymbol{p} & :=\left(P_{1}, P_{2}, \ldots, P_{M}\right)^{\mathrm{T}} \\
& :=\left(E\left[\left|x_{1, l}\right|^{2}\right], E\left[\left|x_{2, l}\right|^{2}\right], \ldots, E\left[\left|x_{M, l}\right|^{2}\right]\right)^{\mathrm{T}} \in \mathbb{R}_{+}^{M}
\end{aligned}
$$

corresponds to precipitation profile along the elevation axis. 


\section{Conventional Methods: Linear BeAmForming}

The beamforming is a reconstruction problem of $\boldsymbol{x}_{l}$ in (10). Major beamforming methods [9]-[19] estimate $\boldsymbol{x}_{l}$ by multiplying complex weights $\boldsymbol{w}_{m} \in \mathbb{C}^{N}(m=1,2, \ldots, M)$ and $\boldsymbol{y}_{l}$ as

$$
\begin{aligned}
\widehat{\boldsymbol{x}}_{l} & :=\left(\widehat{x}_{1, l}, \widehat{x}_{2, l}, \ldots, \widehat{x}_{M, l}\right)^{\mathrm{T}} \\
& :=\left(\boldsymbol{w}_{1}^{\mathrm{H}} \boldsymbol{y}_{l}, \boldsymbol{w}_{2}^{\mathrm{H}} \boldsymbol{y}_{l}, \ldots, \boldsymbol{w}_{M}^{\mathrm{H}} \boldsymbol{y}_{l}\right)^{\mathrm{T}}=\boldsymbol{W} \boldsymbol{y}_{l} .
\end{aligned}
$$

In this paper, we call the methodology based on (11) the linear beamforming. Note that the least squares (LS) method

$$
\widehat{\boldsymbol{x}}_{\mathrm{LS}, l}:=\boldsymbol{W}_{\mathrm{LS}} \boldsymbol{y}_{l}:=\boldsymbol{A}^{\dagger} \boldsymbol{y}_{l}
$$

does not necessarily work well, even if $N \geq M$, since $\boldsymbol{A}$ is illconditioned when $\Delta \theta$ is smaller than the antenna beamwidth that is determined by the antenna size, where $\boldsymbol{A}^{\dagger} \in \mathbb{C}^{M \times N}$ is the Moore-Penrose pseudoinverse of $\boldsymbol{A}$. In the following, we explain three linear methods, Fourier (FR) beamforming [11], Capon (CP) beamforming [12], and MMSE beamforming [19].

\section{A. FR Beamforming}

FR beamforming [11] is the most basic linear method and its complex weight vector is defined by

$$
\boldsymbol{w}_{\mathrm{FR}, m}:=\frac{\boldsymbol{a}_{m}}{N}
$$

independently of $\boldsymbol{y}_{l}$. The vector $\boldsymbol{w}_{\mathrm{FR}, m}$ is equal to a matched filter that maximizes the signal-to-noise ratio $\frac{E\left[\left|x_{m, l} \boldsymbol{w}_{m}^{\mathrm{H}} \boldsymbol{a}_{m}\right|^{2}\right]}{E\left[\left|\boldsymbol{w}_{m}^{\mathrm{H}} \boldsymbol{\varepsilon}_{l}\right|^{2}\right]}$. If $N \geq M$ and there exists some positive integer $n$ satisfying $\forall m \frac{d}{\lambda}\left(\sin \theta_{m+1}-\sin \theta_{m}\right) \approx \frac{n}{N}$, then FR beamforming can reconstruct the signal $\boldsymbol{x}_{l}$ in the manner of the inverse DFT, but this ideal condition is usually not met. In the typical case, the average signal power density $P_{m}$ is often overestimated [19]. To explain this fact, we derive the observation model as in (9) from (10) by redefining $K(\leq M)$ as the number of the subintervals $\left[\theta_{m}-\frac{\Delta \theta}{2}, \theta_{m}+\frac{\Delta \theta}{2}\right]$ in which scatterers (raindrops) exist, and $\theta_{\kappa}^{\star}$ as the center of such a subinterval. Then, from

$\widehat{x}_{\mathrm{FR}, m, l}:=\frac{\boldsymbol{a}_{m}^{\mathrm{H}}}{N} \boldsymbol{y}_{l}=x_{m, l}+\frac{1}{N} \sum_{\theta_{\kappa}^{\star} \neq \theta_{m}} x_{\kappa, l}^{\star} \boldsymbol{a}_{m}^{\mathrm{H}} \boldsymbol{a}\left(\theta_{\kappa}^{\star}\right)+\frac{\boldsymbol{a}_{m}^{\mathrm{H}} \varepsilon_{l}}{N}$,

the signal power $P_{m}=E\left[\left|x_{m, l}\right|^{2}\right]$ is overestimated by (8):

$$
\widehat{P}_{\mathrm{FR}, m}=\frac{1}{L} \sum_{l=1}^{L}\left|\widehat{x}_{\mathrm{FR}, m, l}\right|^{2} \gg E\left[\left|x_{m, l}\right|^{2}\right]+\frac{\sigma_{\varepsilon}^{2}}{N},
$$

for many elevation angles $\theta_{m}$ due to the influence of sidelobes, i.e., the influence of $\theta_{\kappa}^{\star}\left(\neq \theta_{m}\right)$ satisfying $\left|\boldsymbol{a}_{m}^{\mathrm{H}} \boldsymbol{a}\left(\theta_{\kappa}^{\star}\right)\right| \not \approx 0$.

\section{B. CP Beamforming}

CP beamforming [12], that is also known as MVDR beamforming [18], is a data-dependent method, differently from FR beamforming. To avoid the overestimation, $\mathrm{CP}$ beamforming minimizes the signal power $\frac{1}{L} \sum_{l=1}^{L}\left|\widehat{x}_{m, l}\right|^{2}=\boldsymbol{w}_{m}^{\mathrm{H}} \widehat{\boldsymbol{R}}_{y} \boldsymbol{w}_{m}$ under the condition $\boldsymbol{w}_{m}^{\mathrm{H}} \boldsymbol{a}_{m}=1$, where $\widehat{\boldsymbol{R}}_{\boldsymbol{y}}:=\frac{1}{L} \sum_{l=1}^{L} \boldsymbol{y}_{l} \boldsymbol{y}_{l}^{\mathrm{H}} \in$ $\mathbb{C}^{N \times N}$ denotes the sample covariance matrix of the zero-mean random vector $\boldsymbol{y}_{l}$. The weight vector $\boldsymbol{w}_{\mathrm{CP}, m}$ is defined as the solution to the following optimization problem

$$
\underset{\boldsymbol{w}_{m}}{\operatorname{minimize}} \boldsymbol{w}_{m}^{\mathrm{H}} \widehat{\boldsymbol{R}}_{\boldsymbol{y}} \boldsymbol{w}_{m} \quad \text { subject to } \boldsymbol{w}_{m}^{\mathrm{H}} \boldsymbol{a}_{m}=1,
$$

and given by

$$
\boldsymbol{w}_{\mathrm{CP}, m}:=\frac{\widehat{\boldsymbol{R}}_{\boldsymbol{y}}^{-1} \boldsymbol{a}_{m}}{\boldsymbol{a}_{m}^{\mathrm{H}} \widehat{\boldsymbol{R}}_{\boldsymbol{y}}^{-1} \boldsymbol{a}_{m}}
$$

if $L \geq N$ (strictly speaking, if $\operatorname{rank}\left(\widehat{\boldsymbol{R}}_{\boldsymbol{y}}\right)=N$ ). In particular, if $N \geq K+1$ and $L$ is sufficiently large, then we have

$$
\widehat{P}_{\mathrm{CP}, m}:=\frac{1}{L} \sum_{l=1}^{L}\left|\widehat{x}_{\mathrm{CR}, m, l}\right|^{2} \approx E\left[\left|x_{m, l}\right|^{2}\right]+\sigma_{\varepsilon}^{2}\left\|\boldsymbol{w}_{\mathrm{CP}, m}\right\|_{2}^{2}
$$

for all $m$ since $\forall \theta_{\kappa}^{\star} \neq \theta_{m}\left|\boldsymbol{w}_{\mathrm{CP}, m}^{\mathrm{H}} \boldsymbol{a}\left(\theta_{\kappa}^{\star}\right)\right| \approx 0$ holds. However, if $L$ is not large, each average signal power density is underestimated [19]. Moreover if $L<N, \widehat{\boldsymbol{R}}_{\boldsymbol{y}}^{-1}$ cannot be computed.

\section{MMSE Beamforming}

MMSE beamforming [19] was developed to robustly reconstruct the signal $\boldsymbol{x}_{l}$ in case of small $L$. This method approximately solves the following optimization problem

$$
\underset{\boldsymbol{w}_{m}}{\operatorname{minimize}} E\left[\left|x_{m, l}-\boldsymbol{w}_{m}^{\mathrm{H}} \boldsymbol{y}_{l}\right|^{2}\right] \quad \text { subject to } \boldsymbol{w}_{m}^{\mathrm{H}} \boldsymbol{a}_{m}=1 \text {. }
$$

Suppose $E\left[x_{m_{1}, l} \bar{x}_{m_{2}, l}\right]=E\left[x_{m_{1}, l}\right] E\left[\bar{x}_{m_{2}, l}\right]=0\left(m_{1} \neq m_{2}\right)$. Then the exact solution to the optimization problem is given by

$$
\boldsymbol{w}_{\mathrm{MMSE}, m}:=\frac{\boldsymbol{R}_{\boldsymbol{y}}^{-1} \boldsymbol{a}_{m}}{\boldsymbol{a}_{m}^{\mathrm{H}} \boldsymbol{R}_{\boldsymbol{y}}^{-1} \boldsymbol{a}_{m}},
$$

where $\boldsymbol{R}_{\boldsymbol{y}}:=E\left[\boldsymbol{y}_{l} \boldsymbol{y}_{l}^{\mathrm{H}}\right]$ is the covariance matrix of $\boldsymbol{y}_{l}$. By using the covariance matrix $\boldsymbol{R}_{\boldsymbol{x}}:=E\left[\boldsymbol{x}_{l} \boldsymbol{x}_{l}^{\mathrm{H}}\right]$ of $\boldsymbol{x}_{l}, \boldsymbol{R}_{\boldsymbol{y}}$ is expressed as $\boldsymbol{R}_{\boldsymbol{y}}=\boldsymbol{A} \boldsymbol{R}_{\boldsymbol{x}} \boldsymbol{A}^{\mathrm{H}}+\sigma_{\varepsilon}^{2} \boldsymbol{I}_{N}$. Moreover, $\boldsymbol{R}_{\boldsymbol{x}}=\operatorname{diag}(\boldsymbol{p})$ is approximated by $\widehat{\boldsymbol{R}}_{\boldsymbol{x}} \odot \boldsymbol{I}_{M}:=\left(\frac{1}{L} \sum_{l=1}^{L} \boldsymbol{x}_{l} \boldsymbol{x}_{l}^{\mathrm{H}}\right) \odot \boldsymbol{I}_{M}$, where $\odot$ denotes the Hadamard product. As a result, the weight vector $\boldsymbol{w}_{\mathrm{MMSE}, m}$ in (15) can be approximated, from the initial estimate $\widehat{\boldsymbol{x}}_{\mathrm{MMSE}, l}^{(0)}=\widehat{\boldsymbol{x}}_{\mathrm{FR}, l}=\boldsymbol{A}^{\mathrm{H}} \boldsymbol{y}_{l} / N$, by iteratively computing

$$
\begin{aligned}
\boldsymbol{R}_{\boldsymbol{x}}^{(i)} & =\left(\frac{1}{L} \sum_{l=1}^{L} \widehat{\boldsymbol{x}}_{\mathrm{MMSE}, l}^{(i)} \widehat{\boldsymbol{x}}_{\mathrm{MMSE}, l}^{(i) \mathrm{H}}\right) \odot \boldsymbol{I}_{M} \\
\boldsymbol{R}_{\boldsymbol{y}}^{(i)} & =\boldsymbol{A} \boldsymbol{R}_{\boldsymbol{x}}^{(i)} \boldsymbol{A}^{\mathrm{H}}+\sigma_{\varepsilon}^{2} \boldsymbol{I}_{N} \\
\boldsymbol{w}_{\mathrm{MMSE}, m}^{(i+1)} & =\frac{\boldsymbol{R}_{\boldsymbol{y}}^{(i)-1} \boldsymbol{a}_{m}}{\boldsymbol{a}_{m}^{\mathrm{H}} \boldsymbol{R}_{\boldsymbol{y}}^{(i)-1} \boldsymbol{a}_{m}} \quad(m=1,2, \ldots, M) \\
\widehat{\boldsymbol{x}}_{\mathrm{MMSE}, l}^{(i+1)} & =\boldsymbol{W}_{\mathrm{MMSE}}^{(i+1)} \boldsymbol{y}_{l} \quad(l=1,2, \ldots, L)
\end{aligned}
$$

for $i \geq 0$ until $\eta^{(i+1)}=\sum_{m=1}^{M} \frac{\sum_{l=1}^{L}\left|\widehat{x}_{\text {MMSE }, m, l}^{(i+1)}-\widehat{x}_{\text {MMSE }, m, l}^{(i)}\right|^{2}}{M \sum_{l=1}^{L}\left|\widehat{x}_{\text {MMSE }, m, l}^{(i)}\right|^{2}}$ becomes sufficiently small. In this method, even for small $L$, $\boldsymbol{R}_{\boldsymbol{y}}$ and $\boldsymbol{x}_{l}$ are stably estimated. However, if $K$ is close to $N$ or larger than $N$, then the estimation accuracy degrades, i.e., fine fluctuations of the average signal power densities cannot be captured, because each vector $\boldsymbol{w}_{m}$ can only create at most $N-1$ null directions and the influence of the sidelobes occurs.

\section{Nonlinear BeAmforming Via ConveX OPTIMIZATION BASED ON GROUP-SPARSITIES}

In this section, we propose a nonlinear beamforming method based on convex optimization. First, we gather $\boldsymbol{x}_{l}$ and $\boldsymbol{y}_{l}$ into $\boldsymbol{X}:=\left[\begin{array}{llll}\boldsymbol{x}_{1} & \boldsymbol{x}_{2} & \cdots & \boldsymbol{x}_{L}\end{array}\right]=\left[\begin{array}{lllll}\widetilde{\boldsymbol{x}}_{1} & \widetilde{\boldsymbol{x}}_{2} & \cdots & \widetilde{\boldsymbol{x}}_{M}\end{array}\right]^{\mathrm{T}} \in \mathbb{C}^{M \times L}$ and $\boldsymbol{Y}:=\left[\begin{array}{llll}\boldsymbol{y}_{1} & \boldsymbol{y}_{2} & \ldots & \boldsymbol{y}_{L}\end{array}\right] \in \mathbb{C}^{N \times L}$, respectively, where $\widetilde{\boldsymbol{x}}_{m}:=$ $\left(x_{m, 1}, x_{m, 2}, \ldots, x_{m, L}\right)^{\mathrm{T}} \in \mathbb{C}^{L}$. Then the beamforming can be 
translated into a reconstruction problem of $\boldsymbol{X}$ from $\boldsymbol{Y}$, and the data-fidelity for the measurements $\boldsymbol{y}_{l}$ in (10) is evaluated by

$$
\sum_{l=1}^{L}\left\|\boldsymbol{y}_{l}-\boldsymbol{A} \boldsymbol{x}_{l}\right\|_{2}^{2}=\|\boldsymbol{Y}-\boldsymbol{A} \boldsymbol{X}\|_{\mathrm{F}}^{2}=\left\|\boldsymbol{Y}^{\mathrm{T}}-\boldsymbol{X}^{\mathrm{T}} \boldsymbol{A}^{\mathrm{T}}\right\|_{\mathrm{F}}^{2}
$$

For each discrete-time backscattered signal $\widetilde{\boldsymbol{x}}_{m}$ at $\theta_{m}$, define the normalized DFT coefficients $u_{k, m} \in \mathbb{C}(k=1,2, \ldots, L)$ as (5). Let $\boldsymbol{F}:=\frac{1}{\sqrt{L}}\left[\begin{array}{llll}\boldsymbol{f}_{-\frac{L}{2}} & \boldsymbol{f}_{-\frac{L}{2}+1} & \cdots & \boldsymbol{f}_{\frac{L}{2}-1}\end{array}\right]^{\mathrm{T}} \in \mathbb{C}^{L \times L}$ be the normalized discrete Fourier transform matrix, where $f_{i}:=$ $\left(1, e^{-j \frac{2 \pi i}{L}}, e^{-j \frac{4 \pi i}{L}}, \ldots, e^{-j \frac{2(L-1) \pi i}{L}}\right)^{\mathrm{T}} \in \mathbb{C}^{L}$. By using $\boldsymbol{F}$, the DFT coefficient vector $\boldsymbol{u}_{m}:=\left(u_{1, m}, u_{2, m}, \ldots, u_{L, m}\right)^{\mathrm{T}} \in \mathbb{C}^{L}$ is expressed as $\boldsymbol{u}_{m}=\boldsymbol{F} \widetilde{\boldsymbol{x}}_{m}(m=1,2, \ldots, M)$. Furthermore, we define the DFT coefficient matrix $\boldsymbol{U}=\left(u_{k, m}\right) \in \mathbb{C}^{L \times M}$ by

$$
\boldsymbol{U}:=\left[\begin{array}{llll}
\boldsymbol{u}_{1} & \boldsymbol{u}_{2} & \cdots & \boldsymbol{u}_{M}
\end{array}\right]=\boldsymbol{F} \boldsymbol{X}^{\mathrm{T}} .
$$

The reconstruction of $\boldsymbol{X}$ and that of $\boldsymbol{U}$ are essentially equivalent due to the unitarity of $\boldsymbol{F}$, and the weather parameters can be computed from $\boldsymbol{U}$ with (8). Thus in this paper, we consider the beamforming as a reconstruction problem of $\boldsymbol{U}$ from $\boldsymbol{Y}$. We have $\boldsymbol{X}^{\mathrm{T}}=\boldsymbol{F}^{\mathrm{H}} \boldsymbol{U}$, and (17) can be transformed into

$\left\|\boldsymbol{Y}^{\mathrm{T}}-\boldsymbol{X}^{\mathrm{T}} \boldsymbol{A}^{\mathrm{T}}\right\|_{\mathrm{F}}^{2}=\left\|\boldsymbol{Y}^{\mathrm{T}}-\boldsymbol{F}^{\mathrm{H}} \boldsymbol{U} \boldsymbol{A}^{\mathrm{T}}\right\|_{\mathrm{F}}^{2}=\left\|\boldsymbol{F} \boldsymbol{Y}^{\mathrm{T}}-\boldsymbol{U} \boldsymbol{A}^{\mathrm{T}}\right\|_{\mathrm{F}}^{2}$

from the unitarity of $\boldsymbol{F}$. In the following, after describing two group-sparsities of $\boldsymbol{U}$, we estimate $\boldsymbol{U}$ by solving convex optimization problems based on (18) and the two group-sparsities (see Footnote 2 for the meaning of the word "group-sparse").

\section{A. Narrow Bandwidth of Each Power Spectral Density}

As mentioned in Section II-A, the power spectral density of the backscattered signal $x(t)$ is proportional to the Doppler frequency distribution. In addition, the Doppler frequency distribution and the Doppler velocity distribution are essentially the same (see (3)). Thus, unless the Doppler velocity width $\sigma_{v}$ is extremely large, the bandwidth of the power spectral density, similar to the Doppler frequency width $\sigma_{f}$, is narrow compared to the sampling frequency $\frac{1}{T}[\mathrm{~Hz}]$. In particular, when the influence of the atmospheric turbulence is large, ${ }^{5}$ the power spectral density can be modeled by a Gaussian function [7], [26], [27]:

$$
S(f)=P q(f)=\frac{P}{\sqrt{2 \pi} \sigma_{f}} e^{-\frac{\left(f-\mu_{f}\right)^{2}}{2 \sigma_{f}^{2}}} .
$$

In this model, the autocorrelation function is exactly given by

$$
R_{x}(\tau)=\int_{-\infty}^{\infty} S(f) e^{j 2 \pi f \tau} \mathrm{d} f=P e^{j 2 \pi \mu_{f} \tau} e^{-2 \pi^{2} \sigma_{f}^{2} \tau^{2}} .
$$

Figure 1 illustrates the relation between $S(f)$ in (2), $S^{\langle\mathrm{d}\rangle}(f)$ in (4), and the periodogram $\left(\left|u_{k}\right|^{2}\right)_{k=1}^{L}$ in (6) using (19) and (20). As shown in Fig. 1, the frequency indices $k$ of large $E\left[\left|u_{k}\right|^{2}\right]$ are gathered in the vicinity of the mean Doppler frequency $\mu_{f}$.

Since $E\left[\left|u_{k}\right|^{2}\right] \approx 0$ implies $u_{k} \approx 0$ and the above discussion holds for every elevation $\theta_{m}$, each DFT coefficient vector $\boldsymbol{u}_{m}$ will be group-sparse by an appropriate non-overlapping group

\footnotetext{
${ }^{5}$ See, e.g., [26] for a detailed relation between the atmospheric turbulence intensity and the Doppler velocity distribution at each elevation angle.
}

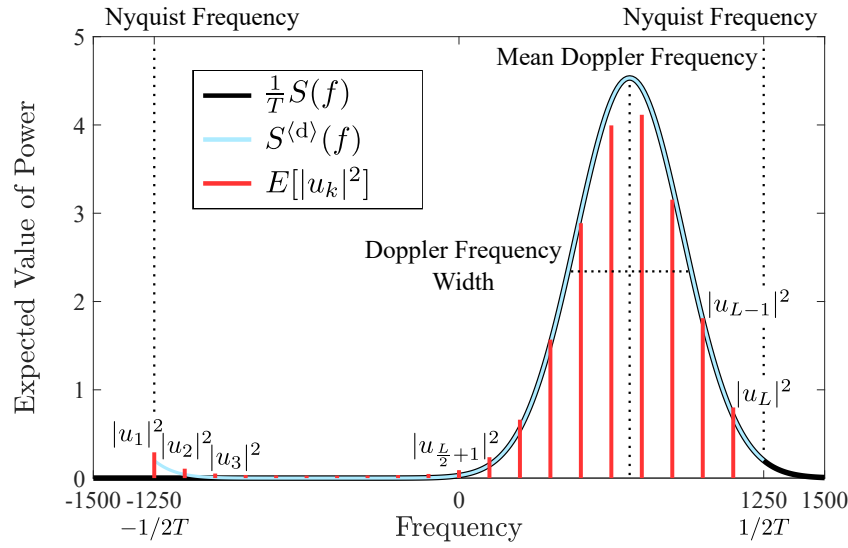

Fig. 1. Relation between the power spectral density $S(f)$ of $x(t)$, the power spectral density $S^{\langle\mathrm{d}\rangle}(f)$ of $x_{l}$, and the periodogram $\left(\left|u_{k}\right|^{2}\right)_{k=1}^{L}$, where the Gaussian function in (19) is used as the Doppler frequency distribution with $P=1, \mu_{f}=700[\mathrm{~Hz}], \sigma_{f}=220[\mathrm{~Hz}], T=4 \times 10^{-4}[\mathrm{~s}]$, and $L=20$. If we set the carrier wavelength to $\lambda=3.181 \times 10^{-2}[\mathrm{~m}]$, then $\sigma_{v} \approx 3.5[\mathrm{~m} / \mathrm{s}]$. Black, blue, and red lines depict $\frac{1}{T} S(f), S^{\langle\mathrm{d}\rangle}(f)$, and $E\left[\left|u_{k}\right|^{2}\right]$, respectively.

partition, but the appropriate partition is unknown in advance. Instead, we divide $\boldsymbol{u}_{m}$ into $L$ overlapping groups of size $b_{\mathrm{f}}$ :

$$
\begin{aligned}
& \boldsymbol{z}_{1, m}^{\langle 1\rangle}:=\left(u_{1, m}, u_{2, m}, \ldots, u_{b_{\mathrm{f}}, m}\right)^{\mathrm{T}} \in \mathbb{C}^{b_{\mathrm{f}}} \\
& \boldsymbol{z}_{2, m}^{\langle 1\rangle}:=\left(u_{2, m}, u_{3, m}, \ldots, u_{b_{\mathrm{f}}+1, m}\right)^{\mathrm{T}} \in \mathbb{C}^{b_{\mathrm{f}}}
\end{aligned}
$$

$$
\left.\begin{array}{c}
\boldsymbol{z}_{L-b_{\mathrm{f}}+1, m}^{\langle 1\rangle}:=\left(u_{L-b_{\mathrm{f}}+1, m}, u_{L-b_{\mathrm{f}}+2, m}, \ldots, u_{L, m}\right)^{\mathrm{T}} \in \mathbb{C}^{b_{\mathrm{f}}} \\
\boldsymbol{z}_{L-b_{\mathrm{f}}+2, m}^{\langle 1\rangle}:=\left(u_{L-b_{\mathrm{f}}+2, m}, \ldots, u_{L, m}, u_{1, m}\right)^{\mathrm{T}} \in \mathbb{C}^{b_{\mathrm{f}}} \\
\vdots \\
\boldsymbol{z}_{L, m}^{\langle 1\rangle}:=\left(u_{L, m}, u_{1, m}, \ldots, u_{b_{\mathrm{f}}-1, m}\right)^{\mathrm{T}} \in \mathbb{C}^{b_{\mathrm{f}}}
\end{array}\right\}
$$

under the periodic boundary condition. Note that we connect $u_{L, m}$ and $u_{1, m}$ in $\boldsymbol{z}_{k, m}^{\langle 1\rangle}\left(k=L-b_{\mathrm{f}}+2, L-b_{\mathrm{f}}+3, \ldots, L\right)$ since the frequency components of the discrete-time signal $\widetilde{\boldsymbol{x}}_{m}$ are wrapped below the Nyquist frequency as shown in Fig. 1. By arranging all $\boldsymbol{z}_{k, m}^{\langle 1\rangle}(k=1,2, \ldots, L$ and $m=1,2, \ldots, M)$, we define a matrix

$$
\boldsymbol{Z}_{1}:=\left[\begin{array}{cccc}
\boldsymbol{z}_{1,1}^{\langle 1\rangle} & \boldsymbol{z}_{1,2}^{\langle 1\rangle} & \cdots & \boldsymbol{z}_{1, M}^{\langle 1\rangle} \\
\boldsymbol{z}_{2,1}^{\langle 1\rangle} & \boldsymbol{z}_{2,2}^{\langle 1\rangle} & \cdots & \boldsymbol{z}_{2, M}^{\langle 1\rangle} \\
\vdots & & & \\
\boldsymbol{z}_{L, 1}^{\langle 1\rangle} & \boldsymbol{z}_{L, 2}^{\langle 1\rangle} & \cdots & \boldsymbol{z}_{L, M}^{\langle 1\rangle}
\end{array}\right] \in \mathbb{C}^{b_{\mathrm{f}} L \times M} .
$$

Then, $\boldsymbol{z}_{k, m}^{\langle 1\rangle} \approx \mathbf{0}$ holds for many $k$ and $m$. Therefore, the matrix $Z_{1}$ will be group-sparse by a non-overlapping partition $G_{1}:=$ $\left(\mathcal{G}_{k, m}^{\langle 1\rangle}\right)$ s.t. $\mathcal{G}_{k, m}^{\langle 1\rangle}:=\left\{\left(b_{\mathrm{f}}(k-1)+i, m\right)\right\}_{i=1}^{b_{\mathrm{f}}}$. Moreover, there is a simple matrix $\boldsymbol{B}_{\mathrm{f}} \in\{0,1\}^{b_{\mathrm{f}} L \times L}$ satisfying $\boldsymbol{B}_{\mathrm{f}} \boldsymbol{U}=\boldsymbol{Z}_{1}$. This property can be evaluated by a weighted group $\ell_{1}$-norm

$$
\left\|\boldsymbol{Z}_{1}\right\|_{1, \boldsymbol{\Xi}_{1}}^{G_{1}}:=\sum_{k=1}^{L} \sum_{m=1}^{M} \xi_{k, m}^{\langle 1\rangle}\left\|\boldsymbol{z}_{k, m}^{\langle 1\rangle}\right\|_{2},
$$

where $\boldsymbol{\Xi}_{1}:=\left(\xi_{k, m}^{\langle 1\rangle}\right) \in \mathbb{R}_{+}^{L \times M}$ is a nonnegative weight matrix. 


\section{B. Similarity between the Adjacent Power Spectral Densities}

In the previous section, we focused on the narrow bandwidth of the power spectral density for each elevation angle $\theta_{m}$. In this section, we explain the similarity between the power spectral densities at adjacent elevation angles $\theta_{m}$ and $\theta_{m+1}$. When $\Delta \theta$ is relatively small, i.e., $M$ is set to a relatively large value to acquire the high-resolution precipitation profile, the mean Doppler velocity $\mu_{v}$ and the Doppler velocity width $\sigma_{v}$ at $\theta_{m}$ and those at $\theta_{m+1}$ are expected to be similar. As a result, the power spectral density at $\theta_{m}$ and that at $\theta_{m+1}$ are similar.

Such a similarity also applies to the adjacent periodograms $\left(\left|u_{k, m}\right|^{2}\right)_{k=1}^{L}$ and $\left(\left|u_{k, m+1}\right|^{2}\right)_{k=1}^{L}$. For a fixed frequency index $k$, if $E\left[\left|u_{k, m}\right|^{2}\right]$ is large, then $E\left[\left|u_{k, m+1}\right|^{2}\right]$ will be also large. If $E\left[\left|u_{k, m}\right|^{2}\right] \approx 0$ holds, then $E\left[\left|u_{k, m+1}\right|^{2}\right] \approx 0$ will also hold. As a result, a vector $\widetilde{\boldsymbol{u}}_{k}:=\left(u_{k, 1}, u_{k, 2}, \ldots, u_{k, M}\right)^{\mathrm{T}} \in \mathbb{C}^{M}$ will be group-sparse by an appropriate non-overlapping group partition, but it is unknown in advance. Instead, as in the previous section, we divide $\widetilde{\boldsymbol{u}}_{k}$ into $M$ overlapping groups of size $b_{\mathrm{e}}$ :

$$
\begin{aligned}
& \boldsymbol{z}_{1, k}^{\langle 2\rangle}:=\left(u_{k, 1}, u_{k, 2}, \ldots, u_{k, b_{\mathrm{e}}}\right)^{\mathrm{T}} \in \mathbb{C}^{b_{\mathrm{e}}} \\
& \boldsymbol{z}_{2, k}^{\langle 2\rangle}:=\left(u_{k, 2}, u_{k, 3}, \ldots, u_{k, b_{\mathrm{e}}+1}\right)^{\mathrm{T}} \in \mathbb{C}^{b_{\mathrm{e}}}
\end{aligned}
$$

$\boldsymbol{z}_{M-b_{\mathrm{e}}+1, k}^{\langle 2\rangle}:=\left(u_{k, M-b_{\mathrm{e}}+1}, u_{k, M-b_{\mathrm{e}}+2}, \ldots, u_{k, M}\right)^{\mathrm{T}} \in \mathbb{C}^{b_{\mathrm{e}}}$ $\boldsymbol{z}_{M-b_{\mathrm{e}}+2, k}^{\langle 2\rangle}:=\left(u_{k, M-b_{\mathrm{e}}+2}, \ldots, u_{k, M}, u_{k, 1}\right)^{\mathrm{T}} \in \mathbb{C}^{b_{\mathrm{e}}}$

$$
\boldsymbol{z}_{M, k}^{\langle 2\rangle}:=\left(u_{k, M}, u_{k, 1}, \ldots, u_{k, b_{\mathrm{e}}-1}\right)^{\mathrm{T}} \in \mathbb{C}^{b_{\mathrm{e}}}
$$

under the periodic boundary condition. By arranging all $\boldsymbol{z}_{m, k}^{\langle 2\rangle}$ ( $m=1,2, \ldots, M$ and $k=1,2, \ldots, L)$, we define a matrix

$$
\boldsymbol{Z}_{2}:=\left[\begin{array}{cccc}
\boldsymbol{z}_{1,1}^{\langle 2\rangle} & \boldsymbol{z}_{1,2}^{\langle 2\rangle} & \cdots & \boldsymbol{z}_{1, L}^{\langle 2\rangle} \\
\boldsymbol{z}_{2,1}^{\langle 2\rangle} & \boldsymbol{z}_{2,2}^{\langle 2\rangle} & \cdots & \boldsymbol{z}_{2, L}^{\langle 2\rangle} \\
\vdots & & & \\
\boldsymbol{z}_{M, 1}^{\langle 2\rangle} & \boldsymbol{z}_{M, 2}^{\langle 2\rangle} & \cdots & \boldsymbol{z}_{M, L}^{\langle 2\rangle}
\end{array}\right] \in \mathbb{C}^{b_{\mathrm{e}} M \times L} .
$$

Then $\boldsymbol{z}_{m, k}^{\langle 2\rangle} \approx \mathbf{0}$ holds for many $m$ and $k$. Therefore, the matrix $Z_{2}$ will be group-sparse by a non-overlapping partition $G_{2}:=$ $\left(\mathcal{G}_{m, k}^{\langle 2\rangle}\right)$ s.t. $\mathcal{G}_{m, k}^{\langle 2\rangle}:=\left\{\left(b_{\mathrm{e}}(m-1)+i, k\right)\right\}_{i=1}^{b_{e}}$, and there exists a simple matrix $\boldsymbol{B}_{\mathrm{e}} \in\{0,1\}^{b_{\mathrm{e}} M \times M}$ satisfying $\boldsymbol{B}_{\mathrm{e}} \boldsymbol{U}^{\mathrm{T}}=\boldsymbol{Z}_{2}$. This property can be evaluated by a weighted group $\ell_{1}$-norm

$$
\left\|\boldsymbol{Z}_{2}\right\|_{1, \boldsymbol{\Xi}_{2}}^{G_{2}}:=\sum_{m=1}^{M-b_{\mathrm{e}}+1} \sum_{k=1}^{L} \xi_{m, k}^{\langle 2\rangle}\left\|\boldsymbol{z}_{m, k}^{\langle 2\rangle}\right\|_{2},
$$

where $\boldsymbol{\Xi}_{2}:=\left(\xi_{m, k}^{\langle 2\rangle}\right) \in \mathbb{R}_{+}^{M \times L}$ is a nonnegative weight matrix s.t. $\xi_{m, k}^{\langle 2\rangle}:=0$ for $m=M-b_{\mathrm{e}}+2, M-b_{\mathrm{e}}+3, \ldots, M$. Note that $E\left[\left|u_{k, M}\right|^{2}\right]$ and $E\left[\left|u_{k, 1}\right|^{2}\right]$ are not necessarily similar, and hence we define the weights $\xi_{m, k}^{\langle 2\rangle}$ as zeros for the vectors $\boldsymbol{z}_{m, k}^{\langle 2\rangle}$ including both $u_{k, M}$ and $u_{k, 1}$ not to evaluate these vectors.

Remark 1 (Difference from Our Previous Work [20]): In our previous work [20], we considered the beamforming as a reconstruction problem of $\boldsymbol{X}$, and used the same group $\ell_{1}$-norm $\left\|\boldsymbol{B}_{\mathrm{f}} \boldsymbol{F} \boldsymbol{X}^{\mathrm{T}}\right\|_{1, \boldsymbol{\Xi}_{1}}^{G_{1}}=\left\|\boldsymbol{B}_{\mathrm{f}} \boldsymbol{U}\right\|_{1, \boldsymbol{\Xi}_{1}}^{G_{1}}=\left\|\boldsymbol{Z}_{1}\right\|_{1, \boldsymbol{\Xi}_{1}}^{G_{1}}$ as in (21) for the narrow bandwidth of each power spectral density. On the other hand, we did not exploit the similarity of the adjacent power spectral densities but use the continuity of precipitation profile, i.e., we assumed that if $\widetilde{\boldsymbol{x}}_{m} \approx \mathbf{0}$, then it is highly possible that $\widetilde{\boldsymbol{x}}_{m+1} \approx \mathbf{0}$. This was evaluated by a weighted group $\ell_{1}$-norm

$$
\begin{aligned}
& \left\|\boldsymbol{B}_{\mathrm{e}} \boldsymbol{X}\right\|_{1, \boldsymbol{\xi}_{0}}^{G_{0}}=\sum_{m=1}^{M-b_{\mathrm{e}}+1} \xi_{m}^{\langle 0\rangle}\left\|\left(\widetilde{\boldsymbol{x}}_{m}^{\mathrm{T}}, \widetilde{\boldsymbol{x}}_{m+1}^{\mathrm{T}}, \ldots, \widetilde{\boldsymbol{x}}_{m+b_{\mathrm{e}}-1}^{\mathrm{T}}\right)^{\mathrm{T}}\right\|_{2} \\
& =\sum_{m=1}^{M-b_{\mathrm{e}}+1} \xi_{m}^{\langle 0\rangle}\left\|\left(\boldsymbol{u}_{m}^{\mathrm{T}}, \boldsymbol{u}_{m+1}^{\mathrm{T}}, \ldots, \boldsymbol{u}_{m+b_{\mathrm{e}}-1}^{\mathrm{T}}\right)^{\mathrm{T}}\right\|_{2} \\
& =\sum_{m=1}^{M-b_{\mathrm{e}}+1} \xi_{m}^{\langle 0\rangle}\left\|\left(\boldsymbol{z}_{m, 1}^{\langle 2\rangle \mathrm{T}}, \boldsymbol{z}_{m, 2}^{\langle 2\rangle \mathrm{T}}, \ldots, \boldsymbol{z}_{m, L}^{\langle 2\rangle \mathrm{T}}\right)^{\mathrm{T}}\right\|_{2}=\left\|\boldsymbol{Z}_{2}\right\|_{1, \boldsymbol{\xi}_{0}}^{G_{0}},
\end{aligned}
$$

where $G_{0}:=\left(\mathcal{G}_{m}^{\langle 0\rangle}\right)$ s.t. $\mathcal{G}_{m}^{\langle 0\rangle}:=\left\{\left(b_{\mathrm{e}}(m-1)+i, k\right)\right\}_{k=1,2, \ldots, L}^{i=1,2, \ldots, b_{\mathrm{e}}}$ and $\boldsymbol{\xi}_{0}:=\left(\xi_{m}^{\langle 0\rangle}\right) \in \mathbb{R}_{+}^{M}$ is some vector s.t. $\xi_{m}^{\langle 0\rangle}=0$ for $m=$ $M-b_{\mathrm{e}}+2, M-b_{\mathrm{e}}+3, \ldots, M$. Therefore, the current work evaluates the group-sparsity of $\boldsymbol{Z}_{2}$ in detail for each frequency by (22), while the previous work roughly evaluated it by collecting all frequency components. In addition, if $\left\|\boldsymbol{Z}_{1}\right\|_{1, \Xi_{1}}^{G_{1}}$ and $\left\|\boldsymbol{Z}_{2}\right\|_{1, \Xi_{2}}^{G_{2}}$ are small, then $\left\|\boldsymbol{Z}_{2}\right\|_{1, \boldsymbol{\xi}_{0}}^{G_{0}}$ will automatically be small. Thus, we do not use our previous cost $\left\|\boldsymbol{Z}_{2}\right\|_{1, \boldsymbol{\xi}_{0}}^{G_{0}}$ in this paper.

\section{The Proposed Nonlinear Beamforming (Formulation I)}

Based on the data-fidelity term in (18) and the two weighted group $\ell_{1}$-norms (21) and (22), we reconstruct $\boldsymbol{U}$ from $\boldsymbol{Y}$ by solving a convex optimization problem

$$
\begin{gathered}
\underset{\boldsymbol{U}, \boldsymbol{Z}_{1}, \boldsymbol{Z}_{2}}{\operatorname{minimize}} \frac{1}{2}\left\|\boldsymbol{F} \boldsymbol{Y}^{\mathrm{T}}-\boldsymbol{U} \boldsymbol{A}^{\mathrm{T}}\right\|_{\mathrm{F}}^{2}+\left\|\boldsymbol{Z}_{1}\right\|_{1, \boldsymbol{\Xi}_{1}}^{G_{1}}+\left\|\boldsymbol{Z}_{2}\right\|_{1, \boldsymbol{\Xi}_{2}}^{G_{2}} \\
\text { subject to } \boldsymbol{Z}_{1}=\boldsymbol{B}_{\mathrm{f}} \boldsymbol{U} \text { and } \boldsymbol{Z}_{2}=\boldsymbol{B}_{\mathrm{e}} \boldsymbol{U}^{\mathrm{T}},
\end{gathered}
$$

with the use of ADMM [21]-[24] (see Appendix for details of ADMM). By defining two convex functions $g$ and $h$ in (40) as

$$
g\left(\boldsymbol{Z}_{1}, \boldsymbol{Z}_{2}\right):=\left\|\boldsymbol{Z}_{1}\right\|_{1, \boldsymbol{\Xi}_{1}}^{G_{1}}+\left\|\boldsymbol{Z}_{2}\right\|_{1, \boldsymbol{\Xi}_{2}}^{G_{2}}
$$

and

$$
h(\boldsymbol{U}):=\frac{1}{2}\left\|\boldsymbol{F} \boldsymbol{Y}^{\mathrm{T}}-\boldsymbol{U} \boldsymbol{A}^{\mathrm{T}}\right\|_{\mathrm{F}}^{2},
$$

the problem in (23) is expressed as an ADMM-form:

$$
\underset{\boldsymbol{U}, \boldsymbol{Z}_{1}, \boldsymbol{Z}_{2}}{\operatorname{minimize}} g\left(\boldsymbol{Z}_{1}, \boldsymbol{Z}_{2}\right)+h(\boldsymbol{U})
$$

subject to $\left(\boldsymbol{Z}_{1}, \boldsymbol{Z}_{2}\right)=\mathcal{L}(\boldsymbol{U}):=\left(\boldsymbol{B}_{\mathrm{f}} \boldsymbol{U}, \boldsymbol{B}_{\mathrm{e}} \circ \mathcal{T}(\boldsymbol{U})\right)$,

where $\mathcal{T}$ is the transpose operator, $\circ$ denotes the composition of two mappings, and $\mathcal{L}: \mathbb{C}^{L \times M} \rightarrow \mathbb{C}^{b_{\mathrm{f}} L \times M} \times \mathbb{C}^{b_{\mathrm{e}} M \times L}$ is an linear mapping that maps $\boldsymbol{U}$ to $\left(\boldsymbol{Z}_{1}, \boldsymbol{Z}_{2}\right)$ with $\boldsymbol{B}_{\mathrm{f}}$ and $\boldsymbol{B}_{\mathrm{e}}$.

The ADMM iterations for (24) are given as follows. On the first line in (41), since the function $g\left(\boldsymbol{Z}_{1}, \boldsymbol{Z}_{2}\right)$ is divided into two parts $\left\|\boldsymbol{Z}_{1}\right\|_{1, \boldsymbol{\Xi}_{1}}^{G_{1}}$ and $\left\|\boldsymbol{Z}_{2}\right\|_{1, \boldsymbol{\Xi}_{2}}^{G_{2}}, \boldsymbol{Z}_{1}$ and $\boldsymbol{Z}_{2}$ are updated by

$$
\left.\begin{array}{l}
\boldsymbol{Z}_{1}^{(i)}=\operatorname{prox}_{\gamma\|\cdot\|_{1, \Xi_{1}}^{G_{1}}}\left(\boldsymbol{B}_{\mathrm{f}} \boldsymbol{U}^{(i)}+\boldsymbol{V}_{1}^{(i)}\right) \\
\boldsymbol{Z}_{2}^{(i)}=\operatorname{prox}_{\gamma\|\cdot\|_{1, \Xi_{2}}^{G_{2}}}\left(\boldsymbol{B}_{\mathrm{e}} \boldsymbol{U}^{(i) \mathrm{T}}+\boldsymbol{V}_{2}^{(i)}\right)
\end{array}\right\}
$$

with the use of the proximity operators of the group $\ell_{1}$-norms in (42), where $\gamma>0$ and $\boldsymbol{V}_{1} \in \mathbb{C}^{b_{\mathrm{f}} L \times M}$ and $\boldsymbol{V}_{2} \in \mathbb{C}^{b_{\mathrm{e}} M \times L}$ are dual variables. Then, on the second line in (41), we have

$$
\left.\begin{array}{c}
\tilde{\boldsymbol{V}}_{1}^{(i)}=\boldsymbol{V}_{1}^{(i)}+\boldsymbol{B}_{\mathrm{f}} \boldsymbol{U}^{(i)}-\boldsymbol{Z}_{1}^{(i)} \\
\tilde{\boldsymbol{V}}_{2}^{(i)}=\boldsymbol{V}_{2}^{(i)}+\boldsymbol{B}_{\mathrm{e}} \boldsymbol{U}^{(i) \mathrm{T}}-\boldsymbol{Z}_{2}^{(i)}
\end{array}\right\} .
$$


On the third line in (41), since $\boldsymbol{U}$ is updated as the solution to a least squares problem, the solution $\boldsymbol{U}^{(i+1)}$ has to satisfy

$$
\begin{aligned}
& \gamma \boldsymbol{U}^{(i+1)} \boldsymbol{A}^{\mathrm{T}} \overline{\boldsymbol{A}}+\mathcal{L}^{*} \circ \mathcal{L}\left(\boldsymbol{U}^{(i+1)}\right) \\
& =\gamma \boldsymbol{F} \boldsymbol{Y}^{\mathrm{T}} \overline{\boldsymbol{A}}+\mathcal{L}^{*}\left(\boldsymbol{Z}_{1}^{(i)}-\widetilde{\boldsymbol{V}}_{1}^{(i)}, \boldsymbol{Z}_{2}^{(i)}-\widetilde{\boldsymbol{V}}_{2}^{(i)}\right),
\end{aligned}
$$

with the adjoint operator $\mathcal{L}^{*}: \mathbb{C}^{b_{\mathrm{f}} L \times M} \times \mathbb{C}^{b_{\mathrm{e}} M \times L} \rightarrow \mathbb{C}^{L \times M}$

$$
\mathcal{L}^{*}\left(\boldsymbol{Z}_{1}, \boldsymbol{Z}_{2}\right):=\boldsymbol{B}_{\mathrm{f}}^{\mathrm{T}} \boldsymbol{Z}_{1}+\mathcal{T}\left(\boldsymbol{B}_{\mathrm{e}}^{\mathrm{T}} \boldsymbol{Z}_{2}\right)=\boldsymbol{B}_{\mathrm{f}}^{\mathrm{T}} \boldsymbol{Z}_{1}+\boldsymbol{Z}_{2}^{\mathrm{T}} \boldsymbol{B}_{\mathrm{e}} .
$$

Moreover, the composite mapping $\mathcal{L}^{*} \circ \mathcal{L}$ can be computed by

$$
\begin{aligned}
\mathcal{L}^{*} \circ \mathcal{L}(\boldsymbol{U}) & =\mathcal{L}^{*}\left(\boldsymbol{B}_{\mathrm{f}} \boldsymbol{U}, \boldsymbol{B}_{\mathrm{e}} \boldsymbol{U}^{\mathrm{T}}\right) \\
& =\boldsymbol{B}_{\mathrm{f}}^{\mathrm{T}} \boldsymbol{B}_{\mathrm{f}} \boldsymbol{U}+\mathcal{T}\left(\boldsymbol{B}_{\mathrm{e}}^{\mathrm{T}} \boldsymbol{B}_{\mathrm{e}} \boldsymbol{U}^{\mathrm{T}}\right) \\
& =b_{\mathrm{f}} \boldsymbol{I}_{L} \boldsymbol{U}+\mathcal{T}\left(b_{\mathrm{e}} \boldsymbol{I}_{M} \boldsymbol{U}^{\mathrm{T}}\right)=\left(b_{\mathrm{f}}+b_{\mathrm{e}}\right) \boldsymbol{U}
\end{aligned}
$$

By substituting (28) into (27), we have

$$
\begin{aligned}
& \boldsymbol{U}^{(i+1)}\left(\gamma \boldsymbol{A}^{\mathrm{T}} \overline{\boldsymbol{A}}+\left(b_{\mathrm{f}}+b_{\mathrm{e}}\right) \boldsymbol{I}_{M}\right) \\
& \quad=\gamma \boldsymbol{F} \boldsymbol{Y}^{\mathrm{T}} \overline{\boldsymbol{A}}+\boldsymbol{B}_{\mathrm{f}}^{\mathrm{T}}\left(\boldsymbol{Z}_{1}^{(i)}-\tilde{\boldsymbol{V}}_{1}^{(i)}\right)+\left(\boldsymbol{Z}_{2}^{(i)}-\tilde{\boldsymbol{V}}_{2}^{(i)}\right)^{\mathrm{T}} \boldsymbol{B}_{\mathrm{e}},
\end{aligned}
$$

and $\boldsymbol{U}^{(i+1)}$ is obtained by applying $\left(\gamma \boldsymbol{A}^{\mathrm{T}} \overline{\boldsymbol{A}}+\left(b_{\mathrm{f}}+b_{\mathrm{e}}\right) \boldsymbol{I}_{M}\right)^{-1}$ to (29) from the right side. Finally, on the fourth line in (42), $\boldsymbol{V}_{1}$ and $\boldsymbol{V}_{2}$ are updated, with the use of $\rho^{(i+1)} \in[0,2]$, by

$$
\left.\begin{array}{l}
\boldsymbol{V}_{1}^{(i+1)}=\widetilde{\boldsymbol{V}}_{1}^{(i)}+\left(\rho^{(i+1)}-1\right)\left(\boldsymbol{B}_{\mathrm{f}} \boldsymbol{U}^{(i+1)}-\boldsymbol{Z}_{1}^{(i)}\right) \\
\boldsymbol{V}_{2}^{(i+1)}=\widetilde{\boldsymbol{V}}_{2}^{(i)}+\left(\rho^{(i+1)}-1\right)\left(\boldsymbol{B}_{\mathrm{e}} \boldsymbol{U}^{(i+1) \mathrm{T}}-\boldsymbol{Z}_{2}^{(i)}\right)
\end{array}\right\} .
$$

By repeating (25), (26), (29) and (30) until a convergence condition is met, the solution to (24) is obtained as an estimate $\widehat{\boldsymbol{U}}$.

\section{The Proposed Nonlinear Beamforming (Formulation II)}

In addition to the optimization problem in (23), we propose another optimization problem in which the relation between $\boldsymbol{U}$ and $\left(\boldsymbol{Z}_{1}, \boldsymbol{Z}_{2}\right)$ is modified to further promote group-sparsities. To increase almost-zero vectors $\boldsymbol{z}_{k, m}^{\langle 1\rangle} \approx \mathbf{0}$ and $\boldsymbol{z}_{m, k}^{\langle 2\rangle} \approx \mathbf{0}$, i.e., to make $\boldsymbol{Z}_{1}$ and $\boldsymbol{Z}_{2}$ more group-sparse, we use the latent group lasso formulation [28]-[31]. Specifically, we propose to solve

$$
\begin{gathered}
\underset{\boldsymbol{U}, \boldsymbol{Z}_{1}, \boldsymbol{Z}_{2}}{\operatorname{minimize}} \frac{1}{2}\left\|\boldsymbol{F} \boldsymbol{Y}^{\mathrm{T}}-\boldsymbol{U} \boldsymbol{A}^{\mathrm{T}}\right\|_{\mathrm{F}}^{2}+\left\|\boldsymbol{Z}_{1}\right\|_{1, \boldsymbol{\Xi}_{1}}^{G_{1}}+\left\|\boldsymbol{Z}_{2}\right\|_{1, \boldsymbol{\Xi}_{2}}^{G_{2}} \\
\text { subject to } \quad \boldsymbol{B}_{\mathrm{f}}^{\mathrm{T}} \boldsymbol{Z}_{1}=\boldsymbol{U} \text { and } \boldsymbol{B}_{\mathrm{e}}^{\mathrm{T}} \boldsymbol{Z}_{2}=\boldsymbol{U}^{\mathrm{T}},
\end{gathered}
$$

where the weight matrix $\boldsymbol{\Xi}_{2}=\left(\xi_{m, k}^{\langle 2\rangle}\right)$ satisfies $\xi_{m, k}^{\langle 2\rangle}=\infty$ for $m=M-b_{\mathrm{e}}+2, M-b_{\mathrm{e}}+3, \ldots, M$, i.e., $\boldsymbol{z}_{m, k}^{\langle 2\rangle}=\mathbf{0}$ has to hold for $m=M-b_{\mathrm{e}}+2, M-b_{\mathrm{e}}+3, \ldots, M$. Differently from the problem in (23), $\boldsymbol{U}$ and $\boldsymbol{U}^{\mathrm{T}}$ in (31) are constructed by applying $\boldsymbol{B}_{\mathrm{f}}^{\mathrm{T}}$ and $\boldsymbol{B}_{\mathrm{e}}^{\mathrm{T}}$ to $\boldsymbol{Z}_{1}$ and $\boldsymbol{Z}_{2}$, respectively. Such a latent group lasso formulation can provide more accurate evaluations of the group-sparsity for fixed-size overlapping groups [30].

To solve the problem in (31) with ADMM, we define five auxiliary variables $\boldsymbol{\Lambda}_{1} \in \mathbb{C}^{b_{\mathrm{f}} L \times M}, \boldsymbol{\Lambda}_{2} \in \mathbb{C}^{b_{\mathrm{e}} M \times L}$, and $\boldsymbol{\Gamma}_{n} \in$ $\mathbb{C}^{L \times M}(n=1,2,3)$. Then, define functions $g$ and $h$ in (40) as

$g\left(\boldsymbol{\Lambda}_{1}, \boldsymbol{\Lambda}_{2}, \boldsymbol{\Gamma}_{1}, \boldsymbol{\Gamma}_{2}, \boldsymbol{\Gamma}_{3}\right):=\left\|\boldsymbol{\Lambda}_{1}\right\|_{1, \boldsymbol{\Xi}_{1}}^{G_{1}}+\left\|\boldsymbol{\Lambda}_{2}\right\|_{1, \boldsymbol{\Xi}_{2}}^{G_{2}}+\iota\left(\boldsymbol{\Gamma}_{1}, \boldsymbol{\Gamma}_{2}, \boldsymbol{\Gamma}_{3}\right)$

and

$$
h\left(\boldsymbol{U}, \boldsymbol{Z}_{1}, \boldsymbol{Z}_{2}\right):=\frac{1}{2}\left\|\boldsymbol{F} \boldsymbol{Y}^{\mathrm{T}}-\boldsymbol{U} \boldsymbol{A}^{\mathrm{T}}\right\|_{\mathrm{F}}^{2},
$$

with the use of an indicator function $\iota$ s.t. $\iota\left(\boldsymbol{\Gamma}_{1}, \boldsymbol{\Gamma}_{2}, \boldsymbol{\Gamma}_{3}\right):=0$ if $\boldsymbol{\Gamma}_{1}=\boldsymbol{\Gamma}_{2}=\boldsymbol{\Gamma}_{3}$ and $\iota\left(\boldsymbol{\Gamma}_{1}, \boldsymbol{\Gamma}_{2}, \boldsymbol{\Gamma}_{3}\right):=\infty$ otherwise. Moreover, by defining an linear mapping $\mathcal{L}$ in (40) as

$$
\mathcal{L}\left(\boldsymbol{U}, \boldsymbol{Z}_{1}, \boldsymbol{Z}_{2}\right):=\left(\boldsymbol{Z}_{1}, \boldsymbol{Z}_{2}, \boldsymbol{U}, \boldsymbol{B}_{\mathrm{f}}^{\mathrm{T}} \boldsymbol{Z}_{1}, \mathcal{T}\left(\boldsymbol{B}_{\mathrm{e}}^{\mathrm{T}} \boldsymbol{Z}_{2}\right)\right),
$$

the problem in (31) is expressed as an ADMM-form:

$$
\underset{\boldsymbol{U}, \boldsymbol{Z}_{1}, \boldsymbol{Z}_{2}, \boldsymbol{\Lambda}_{1}, \boldsymbol{\Lambda}_{2}, \boldsymbol{\Gamma}_{1}, \boldsymbol{\Gamma}_{2}, \boldsymbol{\Gamma}_{3}}{\operatorname{minimiz}} g\left(\boldsymbol{\Lambda}_{1}, \boldsymbol{\Lambda}_{2}, \boldsymbol{\Gamma}_{1}, \boldsymbol{\Gamma}_{2}, \boldsymbol{\Gamma}_{3}\right)+h\left(\boldsymbol{U}, \boldsymbol{Z}_{1}, \boldsymbol{Z}_{2}\right)
$$$$
\text { subject to }\left(\boldsymbol{\Lambda}_{1}, \boldsymbol{\Lambda}_{2}, \boldsymbol{\Gamma}_{1}, \boldsymbol{\Gamma}_{2}, \boldsymbol{\Gamma}_{3}\right)=\mathcal{L}\left(\boldsymbol{U}, \boldsymbol{Z}_{1}, \boldsymbol{Z}_{2}\right) \text {. }
$$

Note that although the function $h\left(\boldsymbol{U}, \boldsymbol{Z}_{1}, \boldsymbol{Z}_{2}\right)$ actually depends only on $\boldsymbol{U}$, we added $\boldsymbol{Z}_{1}$ and $\boldsymbol{Z}_{2}$ as arguments to make it easier to understand that the problem in (32) as the ADMM-from.

The ADMM iterations for (32) are given as follows. On the first line in (41), since $g\left(\boldsymbol{\Lambda}_{1}, \boldsymbol{\Lambda}_{2}, \boldsymbol{\Gamma}_{1}, \boldsymbol{\Gamma}_{2}, \boldsymbol{\Gamma}_{3}\right)$ is divided into three parts $\left\|\boldsymbol{\Lambda}_{1}\right\|_{1, \Xi_{1}}^{G_{1}},\left\|\boldsymbol{\Lambda}_{2}\right\|_{1, \Xi_{2}}^{G_{2}}$, and $\iota\left(\boldsymbol{\Gamma}_{1}, \boldsymbol{\Gamma}_{2}, \boldsymbol{\Gamma}_{3}\right)$, the variables $\boldsymbol{\Lambda}_{1}$ and $\boldsymbol{\Lambda}_{2}$ are updated, with the use of some $\gamma>0$, by

$$
\left.\begin{array}{l}
\boldsymbol{\Lambda}_{1}^{(i)}=\operatorname{prox}_{\gamma\|\cdot\|_{1, \mathbf{\Xi}_{1}}^{G_{1}}}\left(\boldsymbol{Z}_{1}^{(i)}+\boldsymbol{V}_{1}^{(i)}\right) \\
\boldsymbol{\Lambda}_{2}^{(i)}=\operatorname{prox}_{\gamma\|\cdot\|_{1, \mathbf{\Xi}_{2}}^{G_{2}}}\left(\boldsymbol{Z}_{2}^{(i)}+\boldsymbol{V}_{2}^{(i)}\right)
\end{array}\right\},
$$

and $\boldsymbol{\Gamma}_{n}(n=1,2,3)$ are updated, with the use of the proximity operators of the indicator function in (43), by

$\boldsymbol{\Gamma}_{n}^{(i)}=\frac{1}{3}\left(\boldsymbol{U}^{(i)}+\boldsymbol{B}_{\mathrm{f}}^{\mathrm{T}} \boldsymbol{Z}_{1}^{(i)}+\boldsymbol{Z}_{2}^{(i) \mathrm{T}} \boldsymbol{B}_{\mathrm{e}}+\boldsymbol{V}_{3}^{(i)}+\boldsymbol{V}_{4}^{(i)}+\boldsymbol{V}_{5}^{(i)}\right)$,

where $\boldsymbol{V}_{1} \in \mathbb{C}^{b_{\mathrm{f}} L \times M}, \boldsymbol{V}_{2} \in \mathbb{C}^{b_{\mathrm{e}} M \times L}$, and $\boldsymbol{V}_{n} \in \mathbb{C}^{L \times M}$ ( $n=$ $3,4,5)$ are dual variables. On the second line in $(41)$, we have

$$
\left.\begin{array}{rl}
\tilde{\boldsymbol{V}}_{1}^{(i)} & =\boldsymbol{V}_{1}^{(i)}+\boldsymbol{Z}_{1}^{(i)}-\boldsymbol{\Lambda}_{1}^{(i)} \\
\widetilde{\boldsymbol{V}}_{2}^{(i)} & =\boldsymbol{V}_{2}^{(i)}+\boldsymbol{Z}_{2}^{(i)}-\boldsymbol{\Lambda}_{2}^{(i)} \\
\widetilde{\boldsymbol{V}}_{3}^{(i)} & =\boldsymbol{V}_{3}^{(i)}+\boldsymbol{U}^{(i)}-\boldsymbol{\Gamma}_{1}^{(i)} \\
\tilde{\boldsymbol{V}}_{4}^{(i)} & =\boldsymbol{V}_{4}^{(i)}+\boldsymbol{B}_{\mathrm{f}}^{\mathrm{T}} \boldsymbol{Z}_{1}^{(i)}-\boldsymbol{\Gamma}_{2}^{(i)} \\
\widetilde{\boldsymbol{V}}_{5}^{(i)} & =\boldsymbol{V}_{5}^{(i)}+\boldsymbol{Z}_{2}^{(i) \mathrm{T}} \boldsymbol{B}_{\mathrm{e}}-\boldsymbol{\Gamma}_{3}^{(i)}
\end{array}\right\} .
$$

On the third line in (41), $\boldsymbol{U}, \boldsymbol{Z}_{1}$, and $\boldsymbol{Z}_{2}$ can be updated as the solutions to three different least squares problems since the linear mapping $\mathcal{L}$ does not mix $\boldsymbol{U}, \boldsymbol{Z}_{1}$ and $\boldsymbol{Z}_{2}$. Specifically, from

$$
\mathcal{L}^{*}\left(\boldsymbol{\Lambda}_{1}, \boldsymbol{\Lambda}_{2}, \boldsymbol{\Gamma}_{1}, \boldsymbol{\Gamma}_{2}, \boldsymbol{\Gamma}_{3}\right):=\left(\boldsymbol{\Gamma}_{1}, \boldsymbol{\Lambda}_{1}+\boldsymbol{B}_{\mathrm{f}} \boldsymbol{\Gamma}_{2}, \boldsymbol{\Lambda}_{2}+\boldsymbol{B}_{\mathrm{e}} \boldsymbol{\Gamma}_{3}^{\mathrm{T}}\right),
$$

$\boldsymbol{U}$ is updated by

$$
\boldsymbol{U}^{(i+1)}=\left(\gamma \boldsymbol{F} \boldsymbol{Y}^{\mathrm{T}} \overline{\boldsymbol{A}}+\boldsymbol{\Gamma}_{1}^{(i)}-\widetilde{\boldsymbol{V}}_{3}^{(i)}\right)\left(\gamma \boldsymbol{A}^{\mathrm{T}} \overline{\boldsymbol{A}}+\boldsymbol{I}_{M}\right)^{-1} .
$$

$Z_{1}$ and $Z_{2}$ are respectively updated by

$\boldsymbol{Z}_{1}^{(i+1)}=\left(\boldsymbol{I}_{b_{\mathrm{f}} L}+\boldsymbol{B}_{\mathrm{f}} \boldsymbol{B}_{\mathrm{f}}^{\mathrm{T}}\right)^{-1}\left(\boldsymbol{\Lambda}_{1}^{(i)}-\tilde{\boldsymbol{V}}_{1}^{(i)}+\boldsymbol{B}_{\mathrm{f}}\left(\boldsymbol{\Gamma}_{2}^{(i)}-\tilde{\boldsymbol{V}}_{4}^{(i)}\right)\right)$,

and

$\boldsymbol{Z}_{2}^{(i+1)}=\left(\boldsymbol{I}_{b_{\mathrm{e}} M}+\boldsymbol{B}_{\mathrm{e}} \boldsymbol{B}_{\mathrm{e}}^{\mathrm{T}}\right)^{-1}\left(\boldsymbol{\Lambda}_{2}^{(i)}-\widetilde{\boldsymbol{V}}_{2}^{(i)}+\boldsymbol{B}_{\mathrm{e}}\left(\boldsymbol{\Gamma}_{3}^{(i)}-\widetilde{\boldsymbol{V}}_{5}^{(i)}\right)^{\mathrm{T}}\right)$.

Note that, if we use the matrix inversion lemma [32], [33], then $\left(\boldsymbol{I}_{b_{\mathrm{f}} L}+\boldsymbol{B}_{\mathrm{f}} \boldsymbol{B}_{\mathrm{f}}^{\mathrm{T}}\right)^{-1}$ in (37) can be computed by

$$
\begin{aligned}
\left(\boldsymbol{I}_{b_{\mathrm{f}} L}+\boldsymbol{B}_{\mathrm{f}} \boldsymbol{B}_{\mathrm{f}}^{\mathrm{T}}\right)^{-1} & =\boldsymbol{I}_{b_{\mathrm{f}} L}-\boldsymbol{B}_{\mathrm{f}}\left(\boldsymbol{I}_{L}+\boldsymbol{B}_{\mathrm{f}}^{\mathrm{T}} \boldsymbol{B}_{\mathrm{f}}\right)^{-1} \boldsymbol{B}_{\mathrm{f}}^{\mathrm{T}} \\
& =\boldsymbol{I}_{b_{\mathrm{f}} L}-\frac{1}{b_{\mathrm{f}}+1} \boldsymbol{B}_{\mathrm{f}} \boldsymbol{B}_{\mathrm{f}}^{\mathrm{T}},
\end{aligned}
$$




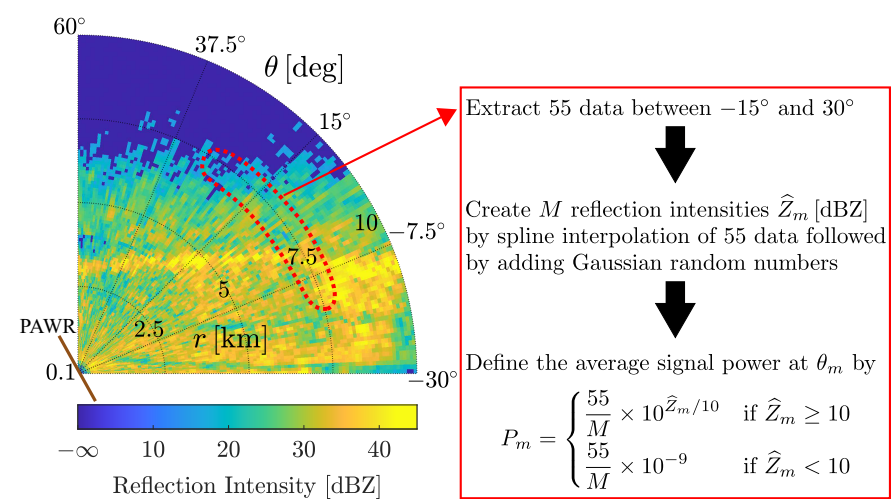

Fig. 2. Real PAWR data observed at Osaka University on March 30th, 2014, and a flowchart on the average signal power density used in our simulations. Note that this real PAWR data is based on the results of FR beamforming.

and $\left(\boldsymbol{I}_{b_{\mathrm{e}} M}+\boldsymbol{B}_{\mathrm{e}} \boldsymbol{B}_{\mathrm{e}}^{\mathrm{T}}\right)^{-1}$ in (38) can also be computed similarly. Finally, on the fourth line in (41), $\boldsymbol{V}_{1}, \boldsymbol{V}_{2}, \boldsymbol{V}_{3}, \boldsymbol{V}_{4}$ and $\boldsymbol{V}_{5}$ are updated, with the use of $\rho^{(i+1)} \in[0,2]$, by

$$
\left.\begin{array}{rl}
\boldsymbol{V}_{1}^{(i+1)} & =\widetilde{\boldsymbol{V}}_{1}^{(i)}+\left(\rho^{(i+1)}-1\right)\left(\boldsymbol{Z}_{1}^{(i+1)}-\boldsymbol{\Lambda}_{1}^{(i)}\right) \\
\boldsymbol{V}_{2}^{(i+1)} & =\widetilde{\boldsymbol{V}}_{2}^{(i)}+\left(\rho^{(i+1)}-1\right)\left(\boldsymbol{Z}_{2}^{(i+1)}-\boldsymbol{\Lambda}_{2}^{(i)}\right) \\
\boldsymbol{V}_{3}^{(i+1)} & =\widetilde{\boldsymbol{V}}_{3}^{(i)}+\left(\rho^{(i+1)}-1\right)\left(\boldsymbol{U}^{(i+1)}-\boldsymbol{\Gamma}_{1}^{(i)}\right) \\
\boldsymbol{V}_{4}^{(i+1)} & =\widetilde{\boldsymbol{V}}_{4}^{(i)}+\left(\rho^{(i+1)}-1\right)\left(\boldsymbol{B}_{\mathrm{f}}^{\mathrm{T}} \boldsymbol{Z}_{1}^{(i+1)}-\boldsymbol{\Gamma}_{2}^{(i)}\right) \\
\boldsymbol{V}_{5}^{(i+1)} & =\widetilde{\boldsymbol{V}}_{5}^{(i)}+\left(\rho^{(i+1)}-1\right)\left(\boldsymbol{Z}_{2}^{(i+1) \mathrm{T}} \boldsymbol{B}_{\mathrm{e}}-\boldsymbol{\Gamma}_{3}^{(i)}\right)
\end{array}\right\}
$$

By repeating (33)-(39) until a convergence condition is met, the solution to (32) is obtained as an estimate $\widehat{U}$.

\section{NUMERICAL Simulations}

\section{A. Simulation Settings}

To show the effectiveness of the proposed nonlinear beamforming, we conducted simulations based on the real reflection intensity, observed by the PAWR at Osaka University, in Fig. 2. We conducted the following simulations on the basis of [34]. At the range $r=7.5[\mathrm{~km}]$, we extracted 55 real reflection intensity data between $\theta_{\min }=-15[\mathrm{deg}]$ and $\theta_{\max }=30[\mathrm{deg}]$. The target reflection intensity $\widehat{Z}_{m}[\mathrm{dBZ}]$ at each $\theta_{m}$ was generated by cubic spline interpolation of these 55 data followed by adding Gaussian random numbers of variance 9 . Note that the random numbers were needed to create the high-resolution precipitation profile. Since the minimum value of the original data in Fig. 2 is 10 [dBZ] in the area where raindrops exist, we defined the average signal power density $P_{m}$ at each $\theta_{m}$ by

$$
P_{m}= \begin{cases}\frac{55}{M} \times 10^{\widehat{Z}_{m} / 10} & \text { if } \widehat{Z}_{m} \geq 10, \\ \frac{55}{M} \times 10^{-9} & \text { if } \widehat{Z}_{m}<10 .\end{cases}
$$

We used $\lambda=31.81[\mathrm{~mm}], d=16.5[\mathrm{~mm}]$, and $T=0.4[\mathrm{~ms}]$. As a result, the Nyquist frequency was $\frac{1}{2 T}=1250[\mathrm{~Hz}]$ and the Nyquist Doppler velocity was $\frac{\lambda}{4 T} \approx 19.88[\mathrm{~m} / \mathrm{s}]$. We used the Gaussian function in (19) as the power spectral density $S_{m}(f)$ at each $\theta_{m}$, and we defined the mean Doppler frequency by

$$
\mu_{f_{m}}=-\frac{2}{\lambda}\left(20 \sin \left(\frac{2 \pi m}{M}\right)+n_{m}\right)[\mathrm{Hz}],
$$

where $n_{m} \in \mathbb{R}$ is a Gaussian random number of variance 1 . The Doppler frequency width at each $\theta_{m}$ was simply fixed to $\sigma_{f_{m}}=\frac{4}{\lambda} \approx 125.7[\mathrm{~Hz}]$ which corresponds to $\sigma_{v_{m}}=2[\mathrm{~m} / \mathrm{s}]$.

The exact autocorrelation function $R_{x_{m}}(\tau)$ of the backscattered signal $x_{m}(t)$ at each $\theta_{m}$ can be computed as in (20), and the covariance matrix of each random vector $\widetilde{\boldsymbol{x}}_{m}$ is given by

$$
\begin{aligned}
& \boldsymbol{R}_{m}:=E\left[\widetilde{\boldsymbol{x}}_{m} \widetilde{\boldsymbol{x}}_{m}^{\mathrm{H}}\right] \\
& =\left[\begin{array}{cccc}
R_{x_{m}}(0) & \overline{R_{x_{m}}(T)} & \ldots & \overline{R_{x_{m}}((L-1) T)} \\
R_{x_{m}}(T) & R_{x_{m}}(0) & \ldots & R_{x_{m}}((L-2) T) \\
\vdots & \vdots & \ddots & \vdots \\
R_{x_{m}}((L-1) T) & R_{x_{m}}((L-2) T) & \ldots & R_{x_{m}}(0)
\end{array}\right] .
\end{aligned}
$$

Based on [7], [35], each target signal $\widetilde{\boldsymbol{x}}_{m}$ was generated from a circularly-symmetric complex Gaussian distribution ${ }^{6}$

$$
\widetilde{q}_{m}(\widetilde{\boldsymbol{x}})=\frac{1}{\pi^{L} \operatorname{det}\left(\boldsymbol{R}_{m}\right)} e^{-\widetilde{\boldsymbol{x}}^{\mathrm{H}} \boldsymbol{R}_{m}^{-1} \widetilde{\boldsymbol{x}}} .
$$

In (10), the variance of the noise $\varepsilon_{l}$ was set to $\sigma_{\varepsilon}^{2}=2.5$.

We set the number of antenna elements to $N=128$. For $M=110$ and $M=166$, we compared the proposed nonlinear beamforming, NL-I in (23) and NL-II in (31), with LS in (12), ${ }^{7}$ FR in (13), and MMSE beamforming in (16), in cases of $L=$ 20 and $L=60$. Note that CP beamforming in (14) cannot be used since $L<N$ holds in both cases. When $M=110$, the number of subintervals where signals exist ${ }^{8}$ was $K=93<N$. On the other hand, when $M=166$, that was $K=142>N$, and hence it is difficult to estimate $\boldsymbol{X}$ or $\boldsymbol{U}$. Moreover, in both cases, the condition number of $\boldsymbol{A}^{\mathrm{H}} \boldsymbol{A}$, denoted by $\operatorname{cn}\left(\boldsymbol{A}^{\mathrm{H}} \boldsymbol{A}\right)$ in Table I, is extremely large, and the beamforming problem based on the observation model in (10) is very ill-conditioned.

The weights of NL-I in (23) were set to $\xi_{k, m}^{\langle 1\rangle}=1.5 \frac{N}{\sqrt{b_{\mathrm{f}}}} \times$ $10^{-3}$ and $\xi_{m, k}^{\langle 2\rangle}=2.5 \frac{N}{\sqrt{b_{\mathrm{e}}}} \times 10^{-4}$, the group size $b_{\mathrm{f}}$ was set to $b_{\mathrm{f}}=3$ for $L=20$ and $b_{\mathrm{f}}=8$ for $L=60$, and the group size $b_{\mathrm{e}}$ was set to $b_{\mathrm{e}}=5$ for $M=110$ and $b_{\mathrm{e}}=7$ for $M=166$. The weights of NL-II in (31) were set to $\xi_{k, m}^{\langle 1\rangle}=6.5 N \sqrt{b_{\mathrm{f}}} \times$ $10^{-4}$ and $\xi_{m, k}^{\langle 2\rangle}=7.5 N \sqrt{b_{\mathrm{e}}} \times 10^{-5}$, the group size $b_{\mathrm{f}}$ was set to $b_{\mathrm{f}}=5$ for $L=20$ and $b_{\mathrm{f}}=13$ for $L=60$, and the group size $b_{\mathrm{e}}$ was set to $b_{\mathrm{e}}=6$ for $M=110$ and $b_{\mathrm{e}}=7$ for $M=166$.

We evaluated the estimate $\widehat{\boldsymbol{X}}$ or $\widehat{\boldsymbol{U}}=\boldsymbol{F} \widehat{\boldsymbol{X}}^{\mathrm{T}}$ of each method by the following normalized mean square error (NMSE).

- NMSE for $x_{m, l}$ or $u_{k, m}$

$$
\begin{aligned}
& \frac{\|\boldsymbol{X}-\widehat{\boldsymbol{X}}\|_{\mathrm{F}}^{2}}{\|\boldsymbol{X}\|_{\mathrm{F}}^{2}}=\frac{\sum_{m=1}^{M} \sum_{l=1}^{L}\left|x_{m, l}-\widehat{x}_{m, l}\right|^{2}}{\sum_{m=1}^{M} \sum_{l=1}^{L}\left|x_{m, l}\right|^{2}} \\
& =\frac{\|\boldsymbol{U}-\widehat{\boldsymbol{U}}\|_{\mathrm{F}}^{2}}{\|\boldsymbol{U}\|_{\mathrm{F}}^{2}}=\frac{\sum_{k=1}^{L} \sum_{m=1}^{M}\left|u_{k, m}-\widehat{u}_{k, m}\right|^{2}}{\sum_{k=1}^{L} \sum_{m=1}^{M}\left|u_{k, m}\right|^{2}} .
\end{aligned}
$$

Moreover, we evaluated the estimated periodograms $\left(\left|\widehat{u}_{k, m}\right|^{2}\right)$ by the following normalized mean absolute errors (NMAEs).

${ }^{6}$ In this model, the variance $V\left[\left|u_{k, m}\right|^{2}\right]:=E\left[\left(\left|u_{k, m}\right|^{2}-E\left[\left|u_{k, m}\right|^{2}\right]\right)^{2}\right]$ of the periodogram satisfies $V\left[\left|u_{k, m}\right|^{2}\right]=\left(E\left[\left|u_{k, m}\right|^{2}\right]\right)^{2}$, which leads to

$$
\lim _{L \rightarrow \infty} V\left[\left|u_{k, m}\right|^{2}\right]=\left(S_{m}^{\langle\mathrm{d}\rangle}\left(\frac{k-1-L / 2}{L T}\right)\right)^{2},
$$

i.e., the variance does not converge to zero when $L$ approaches infinity [36].

${ }^{7}$ To avoid the numerical instability in the computation of the pseudoinverse $\boldsymbol{A}^{\dagger}$, we truncated the singular values of $\boldsymbol{A}$ that are smaller than 0.005 .

${ }^{8}$ We defined $K$ as the number of the indices $m$ s.t. $\widehat{Z}_{m} \geq 10$. 
TABLE I

Averages of the Normalized Errors of the Estimated Signals and the Estimated Periodograms of Each Method in 100 Trials

\begin{tabular}{|c|c|c|c|c|c|c|c|c|}
\hline \multicolumn{3}{|c|}{ imulation Settings } & Evaluation Metric \Method & LS & FR & MMSE & NL-I & NL-II \\
\hline \multirow{4}{*}{$\begin{array}{c}N=128 \\
\lambda=31.81[\mathrm{~mm}] \\
d=16.5[\mathrm{~mm}] \\
T=0.4[\mathrm{~ms}] \\
r=7.5[\mathrm{~km}] \\
c=300000[\mathrm{~km} / \mathrm{s}] \\
\theta_{\min }=-15[\mathrm{deg}] \\
\theta_{\max }=30[\mathrm{deg}]\end{array}$} & \multirow{2}{*}{$\begin{array}{c}\qquad \begin{array}{c}M=110 \\
K=93\end{array} \\
\Delta \theta \approx 0.4091[\mathrm{deg}] \\
\operatorname{cn}\left(\boldsymbol{A}^{\mathrm{H}} \boldsymbol{A}\right)>10^{30} \\
\text { (we used the same } S_{m}(f) \\
\text { for } L=20 \text { and } L=60 \text { ) }\end{array}$} & $L=20$ & $\begin{array}{l}\text { NMSE for } u_{k, m} \\
\text { NMAE for }\left|u_{k, m}\right|^{2} \\
\text { NMAE for } E\left[\left|u_{k, m}\right|^{2}\right] \\
\text { NMAE for } S_{m}^{\langle\mathrm{d}\rangle}\left(\frac{k-1-L / 2}{L T}\right)\end{array}$ & $\begin{array}{l}0.6377 \\
0.9231 \\
0.9185 \\
0.9546\end{array}$ & $\begin{array}{l}1.1060 \\
1.3744 \\
1.6742 \\
1.6975\end{array}$ & $\begin{array}{l}0.4189 \\
0.6372 \\
0.9726 \\
1.0039\end{array}$ & $\begin{array}{l}0.2608 \\
0.5066 \\
0.7223 \\
0.7428 \\
\end{array}$ & $\begin{array}{l}0.2556 \\
0.4931 \\
0.7143 \\
0.7366\end{array}$ \\
\hline & & $L=60$ & $\begin{array}{l}\text { NMSE for } u_{k, m} \\
\text { NMAE for }\left|u_{k, m}\right|^{2} \\
\text { NMAE for } E\left[\left|u_{k, m}\right|^{2}\right] \\
\text { NMAE for } S_{m}^{\langle\mathrm{d}\rangle}\left(\frac{k-1-L / 2}{L T}\right)\end{array}$ & $\begin{array}{l}0.6324 \\
0.9263 \\
0.9222 \\
0.9338\end{array}$ & $\begin{array}{l}1.1057 \\
1.3621 \\
1.6341 \\
1.6407\end{array}$ & $\begin{array}{l}0.4271 \\
0.6549 \\
1.0041 \\
1.0131\end{array}$ & $\begin{array}{l}0.2328 \\
0.4787 \\
0.6845 \\
0.6899 \\
\end{array}$ & $\begin{array}{l}0.2265 \\
0.4669 \\
0.6808 \\
0.6866\end{array}$ \\
\hline & \multirow{2}{*}{$\begin{array}{c}\qquad M=166 \\
K=142 \\
\Delta \theta \approx 0.2711[\mathrm{deg}] \\
\operatorname{cn}\left(\boldsymbol{A}^{\mathrm{H}} \boldsymbol{A}\right)=\infty \\
\text { (we used the same } S_{m}(f) \\
\text { for } L=20 \text { and } L=60 \text { ) }\end{array}$} & $L=20$ & $\begin{array}{l}\text { NMSE for } u_{k, m} \\
\text { NMAE for }\left|u_{k, m}\right|^{2} \\
\text { NMAE for } E\left[\left|u_{k, m}\right|^{2}\right] \\
\text { NMAE for } S_{m}^{\langle\mathrm{d}\rangle}\left(\frac{k-1-L / 2}{L T}\right)\end{array}$ & $\begin{array}{l}0.7324 \\
0.8670 \\
0.8184 \\
0.8639\end{array}$ & $\begin{array}{l}2.1567 \\
2.4415 \\
2.4541 \\
2.4797\end{array}$ & $\begin{array}{l}1.4603 \\
1.6916 \\
1.7487 \\
1.7845\end{array}$ & $\begin{array}{r}\mathbf{0 . 6 0 1 2} \\
0.7816 \\
0.7859 \\
0.8069 \\
\end{array}$ & $\begin{array}{l}0.6078 \\
\mathbf{0 . 7 8 1 3} \\
\mathbf{0 . 7 8 2 8} \\
\mathbf{0 . 8 0 6 0}\end{array}$ \\
\hline & & $L=60$ & $\begin{array}{l}\text { NMSE for } u_{k, m} \\
\text { NMAE for }\left|u_{k, m}\right|^{2} \\
\text { NMAE for } E\left[\left|u_{k, m}\right|^{2}\right] \\
\text { NMAE for } S_{m}^{\langle\mathrm{d}\rangle}\left(\frac{k-1-L / 2}{L T}\right)\end{array}$ & $\begin{array}{l}0.7261 \\
0.8733 \\
0.8292 \\
0.8473\end{array}$ & $\begin{array}{l}2.1812 \\
2.4994 \\
2.5310 \\
2.5390\end{array}$ & $\begin{array}{l}1.5514 \\
1.8467 \\
1.9364 \\
1.9474\end{array}$ & $\begin{array}{l}0.5694 \\
0.7600 \\
0.7487 \\
0.7559 \\
\end{array}$ & $\begin{array}{l}0.5664 \\
0.7556 \\
0.7454 \\
0.7530\end{array}$ \\
\hline
\end{tabular}

- NMAE for $\left|u_{k, m}\right|^{2}$

$$
\frac{\left.\sum_{k=1}^{L} \sum_{m=1}^{M}|| u_{k, m}\right|^{2}-\left|\widehat{u}_{k, m}\right|^{2} \mid}{\sum_{k=1}^{L} \sum_{m=1}^{M}\left|u_{k, m}\right|^{2}} .
$$

- NMAE for $E\left[\left|u_{k, m}\right|^{2}\right]$

$$
\frac{\left.\sum_{k=1}^{L} \sum_{m=1}^{M}\left|E\left[\left|u_{k, m}\right|^{2}\right]-\right| \widehat{u}_{k, m}\right|^{2} \mid}{\sum_{k=1}^{L} \sum_{m=1}^{M} E\left[\left|u_{k, m}\right|^{2}\right]} .
$$

- NMAE for $S_{m}^{\langle\mathrm{d}\rangle}\left(\frac{k-1-L / 2}{L T}\right)$

$$
\frac{\left.\sum_{k=1}^{L} \sum_{m=1}^{M}\left|S_{m}^{\langle\mathrm{d}\rangle}\left(\frac{k-1-L / 2}{L T}\right)-\right| \widehat{u}_{k, m}\right|^{2} \mid}{\sum_{k=1}^{L} \sum_{m=1}^{M} S_{m}^{\langle\mathrm{d}\rangle}\left(\frac{k-1-L / 2}{L T}\right)} .
$$

\section{B. Simulation Results}

Table I summarizes the average, for each situation and each method, of the normalized errors in 100 trials, where the power spectral density $S_{m}(f)$ at each $\theta_{m}$ was fixed throughout all the 100 trials. From Table I, we can see that the proposed methods, NL-I and NL-II, reduced NMSE by $59-64 \%$ from LS, 76-80\% from FR, and 38-47\% from MMSE when $M=110$, while by $17-22 \%$ from LS, $72-74 \%$ from FR, and 58-63\% from MMSE when $M=166$. Thus, the proposed methods greatly improved the estimation accuracy of $\boldsymbol{U}$. In addition, we find that MMSE beamforming estimated $\boldsymbol{X}$ with relatively high accuracy when $M=110$ while the estimation accuracy significantly degraded due to the occurrence of sidelobes when $M=166$.

On the estimated periodograms $\left(\left|\widehat{u}_{k, m}\right|^{2}\right)$, we can see that the proposed methods reduced NMAE for $\left|u_{k, m}\right|^{2}$ by $45-50 \%$ from LS, $63-66 \%$ from FR, and 20-29\% from MMSE when $M=110$, while by $10-13 \%$ from LS, $68-70 \%$ from FR, and $54-59 \%$ from MMSE when $M=166$. Moreover, the proposed methods reduced NMAEs for $E\left[\left|u_{k, m}\right|^{2}\right]$ and $S_{m}^{\langle\mathrm{d}\rangle}\left(\frac{k-1-L / 2}{L T}\right)$ by $21-26 \%$ from LS, $56-58 \%$ from FR, and $26-32 \%$ from MMSE when $M=110$, while by $4-11 \%$ from LS, $67-71 \%$ from FR, and $55-62 \%$ from MMSE when $M=166$. Thus, the proposed methods obtained the most accurate periodograms.
On the number of pulses, all the linear methods, LS, FR, and MMSE, did not necessarily reduce NMSE and NMAEs, even if $L$ was changed from 20 to 60 . On the other hand, the proposed methods, NL-I and NL-II, could reduce NMSE and NMAEs by changing $L$ from 20 to 60 . This is because, as $L$ increases, $E\left[\left|u_{k, m}\right|^{2}\right]$ approaches $S_{m}^{\langle\mathrm{d}\rangle}\left(\frac{k-1-L / 2}{L T}\right)$ and $\boldsymbol{U}$ becomes more group-sparse, which is ideal for the proposed methods.

Figures 3, 4, 5, and 6 show examples of beamforming results for $(M, L)=(110,20),(110,60),(166,20),(166,60)$, where (a) shows the power spectral densities, (b) shows the expected values of periodograms, (c) shows target periodograms, and (d), (e), (f), (g) and (h) show estimated periodograms by LS, FR, MMSE, NL-I and NL-II beamforming, respectively. From Figs. 3(a)-3(c), 4(a)-4(c), 5(a)-5(c), and 6(a)-6(c), we can see that $E\left[\left|u_{k, m}\right|^{2}\right]$ in (b) approached $S_{m}^{\langle\mathrm{d}\rangle}\left(\frac{k-1-L / 2}{L T}\right)$ in (a), and the target periodograms $\left(\left|u_{k, m}\right|^{2}\right)$ in (c) became more groupsparse as the number $L$ of pulses increased from 20 to 60 .

When $M=110$, from Figs. 3(d) and 4(d), LS beamforming diffused the energy of the backscattered signals to all elevation angles, and these results cannot be used for the weather observation. From Figs. 3(e) and 4(e), FR beamforming failed in the estimation particularly from 23 [deg] to 27 [deg] since the signal power should be almost 0 in this interval. From Figs. 3(f) and 4(f), MMSE beamforming obtained the periodograms with relatively high accuracy but the energy of the target signals was diffused mainly in the elevation direction. On the other hand, from Figs. 3(g), 3(h), 4(g), and 4(h), we can confirm that the proposed nonlinear methods, NL-I and NL-II, could obtain the accurate periodograms with less energy diffusion. Though the results by NL-I and NL-II were very similar, NL-II could give the slightly higher-resolution periodograms than NL-I.

When $M=166$, each signal power $P_{m}$ decreases compared to the case of $M=110$ since the width $\Delta \theta$ of each subinterval $\left[\theta_{m}-\frac{\Delta \theta}{2}, \theta_{m}+\frac{\Delta \theta}{2}\right]$ becomes narrower as $M$ increases. Hence, if we increase the number $M$ of the subintervals, then not only the number of variables to be estimated increases, but also the signal-to-noise ratio decreases. Even in such a severe situation, 


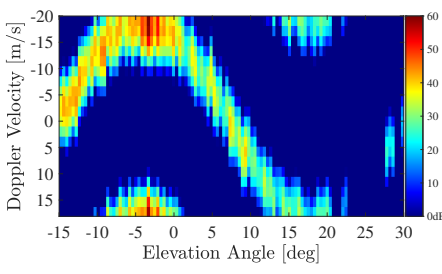

(a)

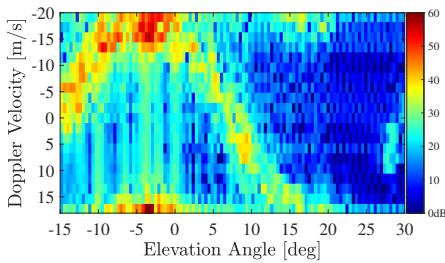

(e)

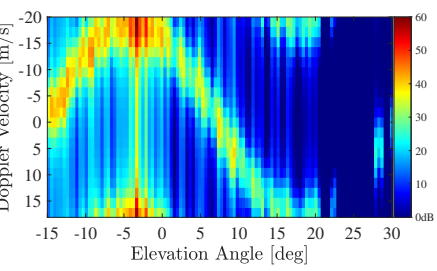

(b)

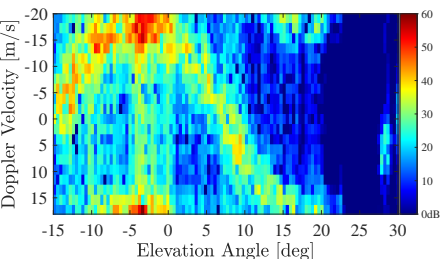

(f)

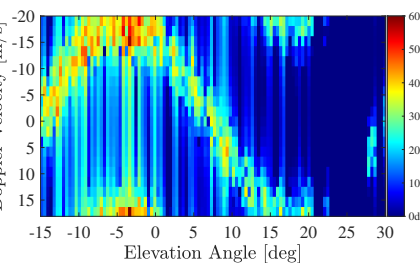

(c)

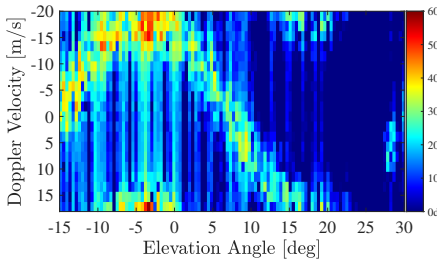

(g)

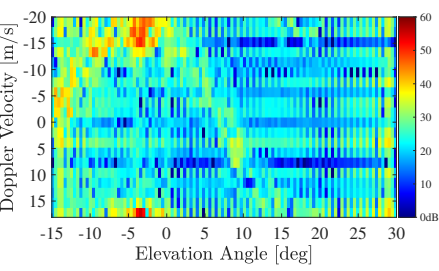

(d)

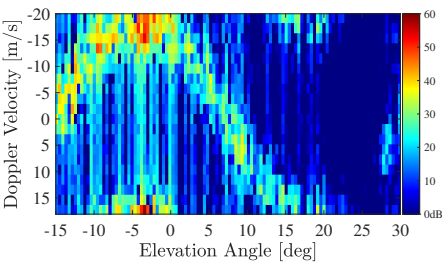

(h)

Fig. 3. Comparison of the power spectral densities, true periodograms, and each beamforming result in case of $M=110$ and $L=20$ : (a) $S_{m}^{\langle\text {d }\rangle}\left(\frac{k-1-L / 2}{L T}\right)$, (b) $E\left[\left|u_{k, m}\right|^{2}\right]$, (c) $\left|u_{k, m}\right|^{2}$, (d) $\left|\widehat{u}_{k, m}\right|^{2}$ by LS, (e) $\left|\widehat{u}_{k, m}\right|^{2}$ by FR, (f) $\left|\widehat{u}_{k, m}\right|^{2}$ by MMSE, (g) $\left|\widehat{u}_{k, m}\right|^{2}$ by NL-I, and (h) $\left|\widehat{u}_{k, m}\right|^{2}$ by NL-II beamforming.

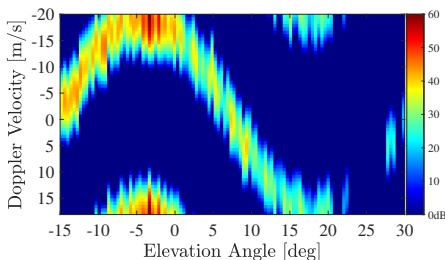

(a)

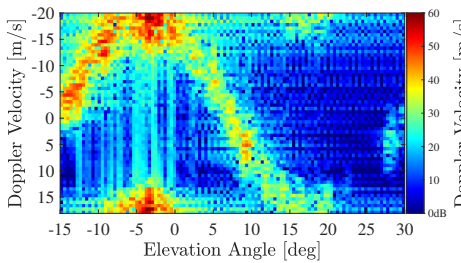

(e)

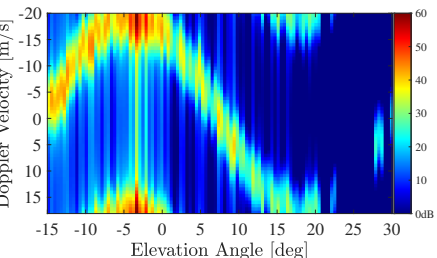

(b)

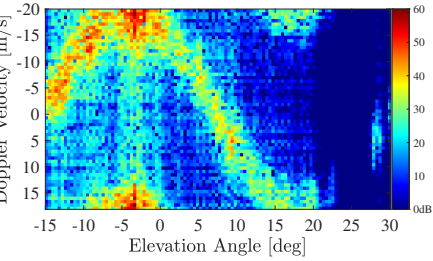

(f)

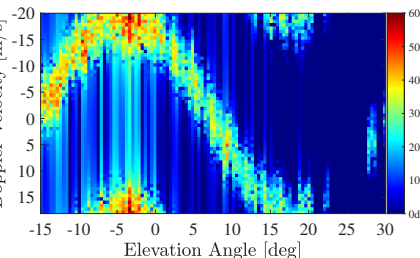

(c)

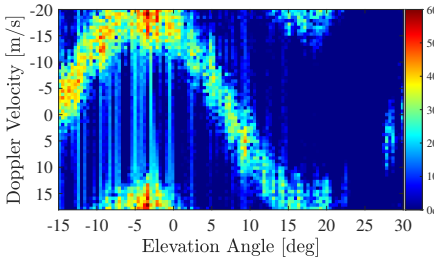

(g)

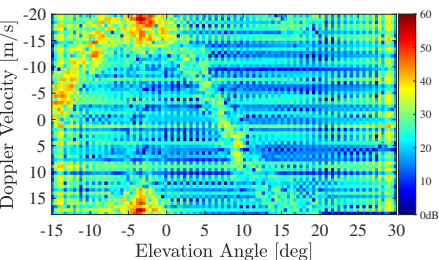

(d)

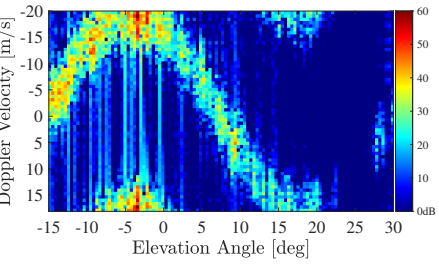

(h)

Fig. 4. Comparison of the power spectral densities, true periodograms, and each beamforming result in case of $M=110$ and $L=60$ : (a) $S_{m}^{\langle\text {d }\rangle}\left(\frac{k-1-L / 2}{L T}\right)$, (b) $E\left[\left|u_{k, m}\right|^{2}\right]$, (c) $\left|u_{k, m}\right|^{2}$, (d) $\left|\widehat{u}_{k, m}\right|^{2}$ by LS, (e) $\left|\widehat{u}_{k, m}\right|^{2}$ by FR, (f) $\left|\widehat{u}_{k, m}\right|^{2}$ by MMSE, (g) $\left|\widehat{u}_{k, m}\right|^{2}$ by NL-I, and (h) $\left|\widehat{u}_{k, m}\right|^{2}$ by NL-II beamforming.

the proposed nonlinear methods, NL-I and NL-II, obtained the most accurate periodograms as shown in Figs. 5(g), 5(h), 6(g), and 6(h). As in the case of $M=110$, NL-I and NL-II obtained the high-resolution periodograms without the energy diffusion, while the conventional linear methods, LS, FR and MMSE, led to the blurring and the overestimation. Thus, the proposed nonlinear beamforming greatly improved the spatial resolution.

\section{CONCLUSION}

In this paper, we proposed a nonlinear beamforming method for a PAWR. Differently from a standard radar which observes point targets, the PAWR receives a lot of backscattered signals from distributed targets, and thus the spatial resolution of the linear beamforming methods is limited. To acquire the highresolution precipitation profile, we used two characteristics of periodograms of the backscattered signals. One is the narrow bandwidth of each periodogram, and the other is the similarity between the adjacent periodograms. Both characteristics were expressed as group-sparsities of the DFT coefficient matrix of the backscattered signals. We proposed to reconstruct the DFT coefficient matrix by minimizing a convex cost function which consists of one data-fidelity term and two group $\ell_{1}$-norms with ADMM. Numerical simulations based on the real data showed that, compared to the linear methods, the proposed nonlinear beamforming obtains the high-resolution precipitation profile.

\section{APPENDIX}

\section{Alternating Direction Method of Multipliers}

Let us consider the following convex optimization problem

$$
\underset{\boldsymbol{x} \in \mathcal{X}, \boldsymbol{z} \in \mathcal{Z}}{\operatorname{minimize}} g(\boldsymbol{z})+h(\boldsymbol{x}) \quad \text { subject to } \boldsymbol{z}=\mathcal{L}(\boldsymbol{x}),
$$

where $\mathcal{X}$ and $\mathcal{Z}$ are finite-dimensional real Hilbert spaces with the standard inner products, $\mathcal{L}: \mathcal{X} \rightarrow \mathcal{Z}$ is a linear mapping, and both functions $g: \mathcal{Z} \rightarrow \mathbb{R} \cup\{\infty\}$ and $h: \mathcal{X} \rightarrow \mathbb{R} \cup\{\infty\}$ are proper, lower semicontinuous, and convex. ${ }^{9}$ The alternating

${ }^{9}$ A function $h: \mathcal{X} \rightarrow \mathbb{R} \cup\{\infty\}$ is called proper, lower semicontinuous, and convex if $\operatorname{dom}(h):=\{\boldsymbol{x} \in \mathcal{X} \mid h(\boldsymbol{x})<\infty\}$ is nonempty, lev $\leq_{\alpha}(h):=$ $\{\boldsymbol{x} \in \mathcal{X} \mid h(\boldsymbol{x}) \leq \alpha\}$ is closed for all $\alpha \in \mathbb{R}$, and $h\left(\beta \boldsymbol{x}_{1}+(1-\beta) \boldsymbol{x}_{2}\right) \leq$ $\beta h\left(\boldsymbol{x}_{1}\right)+(1-\bar{\beta}) h\left(\boldsymbol{x}_{2}\right)$ for all $\boldsymbol{x}_{1}, \boldsymbol{x}_{2} \in \mathcal{X}$ and all $\beta \in(0,1)$, respectively. 


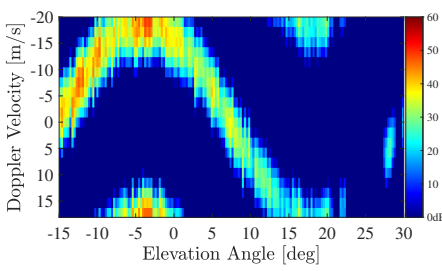

(a)

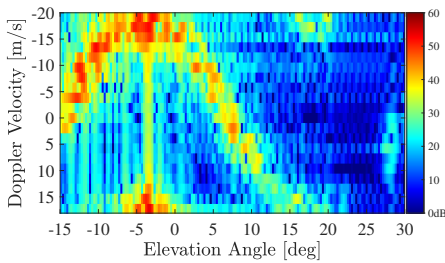

(e)

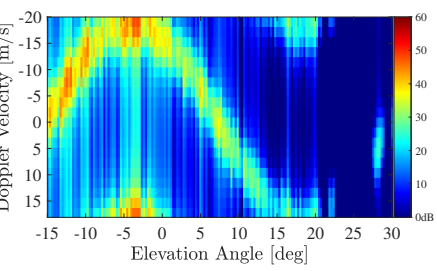

(b)

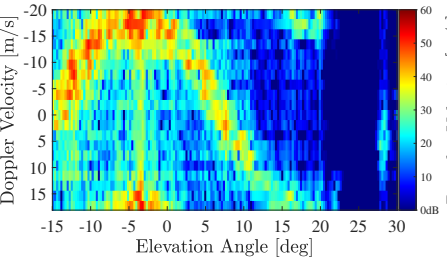

(f)

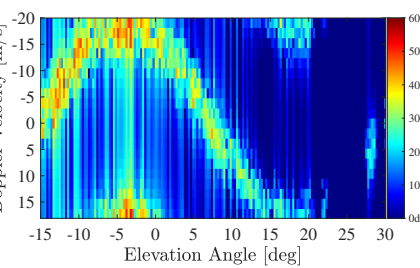

(c)

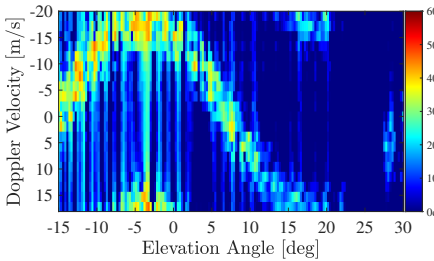

$(\mathrm{g})$

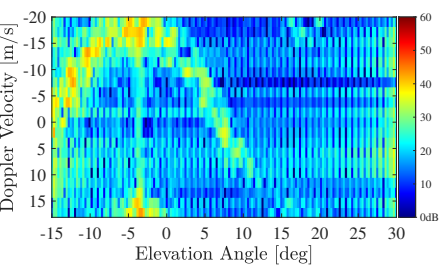

(d)

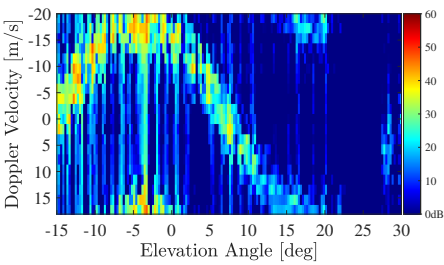

(h)

Fig. 5. Comparison of the power spectral densities, true periodograms, and each beamforming result in case of $M=166$ and $L=20$ : (a) $S_{m}^{\langle\text {d }\rangle}\left(\frac{k-1-L / 2}{L T}\right)$, (b) $E\left[\left|u_{k, m}\right|^{2}\right]$, (c) $\left|u_{k, m}\right|^{2}$, (d) $\left|\widehat{u}_{k, m}\right|^{2}$ by LS, (e) $\left|\widehat{u}_{k, m}\right|^{2}$ by FR, (f) $\left|\widehat{u}_{k, m}\right|^{2}$ by MMSE, (g) $\left|\widehat{u}_{k, m}\right|^{2}$ by NL-I, and (h) $\left|\widehat{u}_{k, m}\right|^{2}$ by NL-II beamforming.

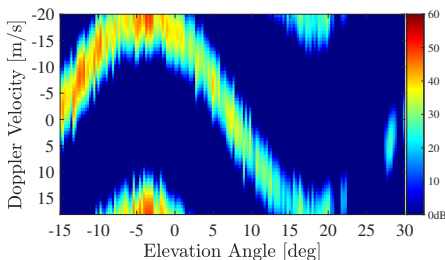

(a)

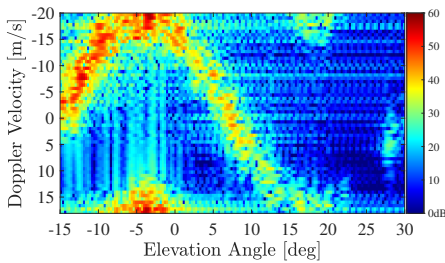

(e)

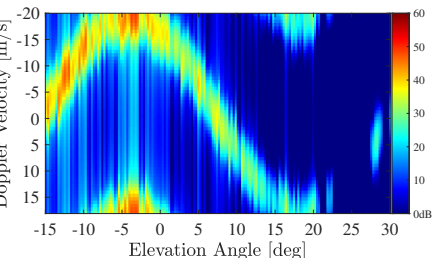

(b)

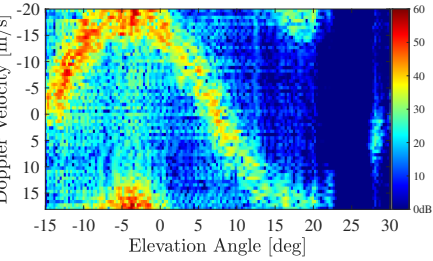

(f)

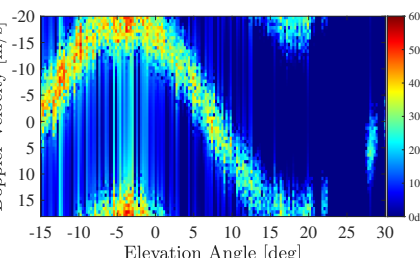

(c)

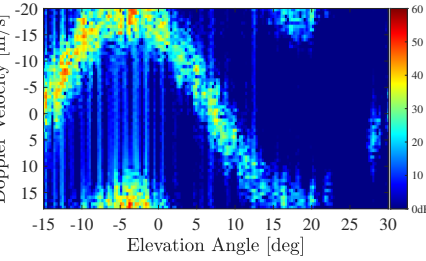

(g)

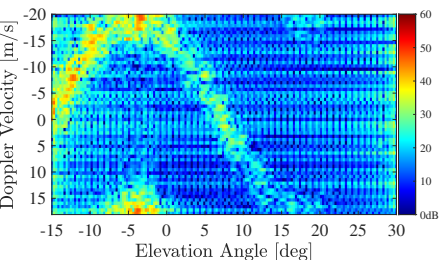

(d)

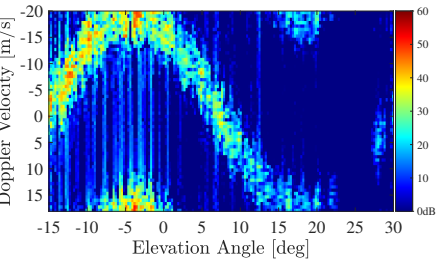

(h)

Fig. 6. Comparison of the power spectral densities, true periodograms, and each beamforming result in case of $M=166$ and $L=60$ : (a) $S_{m}^{\langle\text {d }\rangle}\left(\frac{k-1-L / 2}{L T}\right)$, (b) $E\left[\left|u_{k, m}\right|^{2}\right]$, (c) $\left|u_{k, m}\right|^{2}$, (d) $\left|\widehat{u}_{k, m}\right|^{2}$ by LS, (e) $\left|\widehat{u}_{k, m}\right|^{2}$ by FR, (f) $\left|\widehat{u}_{k, m}\right|^{2}$ by MMSE, (g) $\left|\widehat{u}_{k, m}\right|^{2}$ by NL-I, and (h) $\left|\widehat{u}_{k, m}\right|^{2}$ by NL-II beamforming.

direction method of multipliers (ADMM) [21]-[24] solves the problem in (40), together with the corresponding dual problem, from any $\left(\boldsymbol{x}^{(0)}, \boldsymbol{v}^{(0)}\right) \in \mathcal{X} \times \mathcal{Z}$ by iteratively computing

$$
\begin{aligned}
\boldsymbol{z}^{(i)} & =\operatorname{prox}_{\gamma g}\left(\mathcal{L}\left(\boldsymbol{x}^{(i)}\right)+\boldsymbol{v}^{(i)}\right) \\
\widetilde{\boldsymbol{v}}^{(i)} & =\boldsymbol{v}^{(i)}+\mathcal{L}\left(\boldsymbol{x}^{(i)}\right)-\boldsymbol{z}^{(i)} \\
\boldsymbol{x}^{(i+1)} & =\underset{\boldsymbol{x} \in \mathcal{X}}{\operatorname{argmin}} \gamma h(\boldsymbol{x})+\frac{1}{2}\left\|\mathcal{L}(\boldsymbol{x})-\boldsymbol{z}^{(i)}+\widetilde{\boldsymbol{v}}^{(i)}\right\|_{\mathcal{X}}^{2} \\
\boldsymbol{v}^{(i+1)} & =\widetilde{\boldsymbol{v}}^{(i)}+\left(\rho^{(i+1)}-1\right)\left(\mathcal{L}\left(\boldsymbol{x}^{(i+1)}\right)-\boldsymbol{z}^{(i)}\right)
\end{aligned}
$$

for $i \geq 0$. In (41), $\gamma>0, \boldsymbol{v} \in \mathcal{Z}$ and $\widetilde{\boldsymbol{v}} \in \mathcal{Z}$ are dual variables, $\|\cdot\|_{\mathcal{X}}$ is the Euclidean norm of $\mathcal{X}$ induced by the standard inner

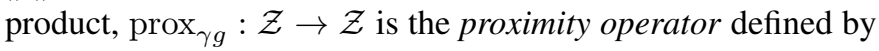

$$
\operatorname{prox}_{\gamma g}(\boldsymbol{\zeta}):=\underset{\boldsymbol{z} \in \mathcal{Z}}{\operatorname{argmin}} \gamma g(\boldsymbol{z})+\frac{1}{2}\|\boldsymbol{\zeta}-\boldsymbol{z}\|_{\mathcal{Z}}^{2},
$$

and $\rho^{(i+1)} \in[0,2]$ satisfies $\sum_{i=0}^{\infty} \rho^{(i+1)}\left(2-\rho^{(i+1)}\right)=\infty$. In particular, if $\rho^{(i+1)}=1$ for all $i$, which is the most commonly used setting, then we can replace $\widetilde{\boldsymbol{v}}^{(i)}$ with $\boldsymbol{v}^{(i+1)}$ and remove the fourth line in (41). The sequence $\left(\boldsymbol{x}^{(i)}, \boldsymbol{z}^{(i)}\right)_{i=0}^{\infty}$ defined by the iterations in (41) converges to a solution to (40). Moreover, the sequences $\left(\boldsymbol{v}^{(i)} / \gamma\right)_{i=0}^{\infty}$ and $\left(\widetilde{\boldsymbol{v}}^{(i)} / \gamma\right)_{i=0}^{\infty}$ both converge to a solution to the dual problem. As described in [24], the overrelaxation setting, i.e., $\rho^{(i+1)}>1$ for all $i$, can accelerate the convergence, compared to the standard setting s.t. $\rho^{(i+1)}=1$.

In the following, we introduce the proximity operators used in this paper. When $g$ is a weighted group $\ell_{1}$-norm with a nonoverlapping group partition $G:=\left(\mathcal{G}_{i}\right)_{i=1}^{n_{G}}$ and a weight vector $\boldsymbol{\xi}:=\left(\xi_{1}, \xi_{2}, \ldots, \xi_{n_{G}}\right)^{\mathrm{T}} \in \mathbb{R}_{+}^{n_{G}}$, from $\gamma g(\boldsymbol{z})+\frac{1}{2}\|\boldsymbol{\zeta}-\boldsymbol{z}\|_{\mathcal{Z}}^{2}=$ $\sum_{i=1}^{n_{G}}\left(\gamma \xi_{i}\left\|\boldsymbol{z}_{\mathcal{G}_{i}}\right\|_{2}+\frac{1}{2}\left\|\boldsymbol{\zeta}_{\mathcal{G}_{i}}-\boldsymbol{z}_{\mathcal{G}_{i}}\right\|_{2}^{2}\right)$, the computation of prox $\operatorname{sig}_{\gamma g}$ is divided into those of $\operatorname{prox}_{\gamma \xi_{i}\|\cdot\|_{2}}$. Specifically, $\operatorname{prox}_{\gamma g}(\boldsymbol{\zeta})=$ $\operatorname{prox}_{\gamma\|\cdot\|_{1, \boldsymbol{\xi}}^{G}}(\boldsymbol{\zeta})$ can be computed for each sub-vector $\boldsymbol{\zeta}_{\mathcal{G}_{i}}$ by

$$
\operatorname{prox}_{\gamma \xi_{i}\|\cdot\|_{2}}\left(\boldsymbol{\zeta}_{\mathcal{G}_{i}}\right)= \begin{cases}\frac{\left\|\boldsymbol{\zeta}_{\mathcal{G}_{i}}\right\|_{2}-\gamma \xi_{i}}{\left\|\boldsymbol{\zeta}_{\mathcal{G}_{i}}\right\|_{2}} \zeta_{\mathcal{G}_{i}} & \text { if }\left\|\boldsymbol{\zeta}_{\mathcal{G}_{i}}\right\|_{2}>\gamma \xi_{i}, \\ 0 & \text { if }\left\|\boldsymbol{\zeta}_{\mathcal{G}_{i}}\right\|_{2} \leq \gamma \xi_{i}\end{cases}
$$

Let $\boldsymbol{\zeta}_{i} \in \mathcal{Z}(i=1,2, \ldots, n)$ be $n$ variables of the same size. Let $\iota$ be the indicator function of the consensus set [37] for the $n$-tuple $\left(\boldsymbol{\zeta}_{1}, \boldsymbol{\zeta}_{2}, \ldots, \boldsymbol{\zeta}_{n}\right)$, i.e., $\iota\left(\boldsymbol{\zeta}_{1}, \boldsymbol{\zeta}_{2}, \ldots, \boldsymbol{\zeta}_{n}\right):=0$ if $\boldsymbol{\zeta}_{1}=$ 
$\boldsymbol{\zeta}_{2}=\cdots=\boldsymbol{\zeta}_{n}$ and $\iota\left(\boldsymbol{\zeta}_{1}, \boldsymbol{\zeta}_{2}, \ldots, \boldsymbol{\zeta}_{n}\right):=\infty$ otherwise. The proximity operators of $\iota$ can be easily computed by

$$
\operatorname{prox}_{\gamma \iota}\left(\boldsymbol{\zeta}_{1}, \boldsymbol{\zeta}_{2}, \ldots, \boldsymbol{\zeta}_{n}\right)=\frac{1}{n}\left(\sum_{i=1}^{n} \boldsymbol{\zeta}_{i}, \sum_{i=1}^{n} \boldsymbol{\zeta}_{i}, \ldots, \sum_{i=1}^{n} \boldsymbol{\zeta}_{i}\right) \text {. }
$$

As can be seen from (43), the proximity operator of the indicator function is equal to the projection onto the consensus set and does not depend on the value of $\gamma$ differently from (42).

\section{REFERENCES}

[1] D. S. Zrnic, J. F. Kimpel, D. E. Forsyth, A. Shapiro, G. Crain, R. Ferek, J. Heimmer, W. Benner, T. J. McNellis, and R. J. Vogt, "Agile-beam phased array radar for weather observations," Bulletin of the American Meteorological Society, vol. 88, no. 11, pp. 1753-1766, Nov. 2007.

[2] D. McLaughlin, D. Pepyne, V. Chandrasekar, B. Philips, J. Kurose, M. Zink, K. Droegemeier, S. Cruz-Pol, F. Junyent, J. Brotzge, D. Westbrook, N. Bharadwaj, Y. Wang, E. Lyons, K. Hondl, Y. Liu, E. Knapp, M. Xue, A. Hopf, K. Kloesel, A. DeFonzo, P. Kollias, K. Brewster, R. Contreras, B. Dolan, T. Djaferis, E. Insanic, S. Frasier, and F. Carr, "Short-wavelength technology and the potential for distributed networks of small radar systems," Bulletin of the American Meteorological Society, vol. 90, no. 12, pp. 1797-1818, Dec. 2009.

[3] B. Isom, R. Palmer, R. Kelley, J. Meier, D. Bodine, M. Yeary, B. L. Cheong, Y. Zhang, T. Y. Yu, and M. I. Biggerstaff, "The atmospheric imaging radar: Simultaneous volumetric observations using a phased array weather radar," Journal of Atmospheric and Oceanic Technology, vol. 30, no. 4, pp. 655-675, Apr. 2013.

[4] D. S. Zrnic, V. M. Melnikov, R. J. Doviak, and R. Palmer, "Scanning strategy for the multifunction phased-array radar to satisfy aviation and meteorological needs," IEEE Geoscience and Remote Sensing Letters, vol. 12, no. 6, pp. 1204-1208, Jun. 2015.

[5] F. Mizutani, T. Ushio, E. Yoshikawa, S. Shimamura, H. Kikuchi, M. Wada, S. Satoh, and T. Iguchi, "Fast-scanning phased-array weather radar with angular imaging technique," IEEE Transactions on Geoscience and Remote Sensing, vol. 56, no. 5, pp. 2664-2673, May 2018.

[6] E. Yoshikawa, T. Ushio, and H. Kikuchi, "A study of comb beam transmission on phased array weather radars," IEEE Transactions on Geoscience and Remote Sensing (Early Access), 11 pares, Oct. 2020.

[7] V. N. Bringi and V. Chandrasekar, Polarimetric Doppler Weather Radar: Principles and Applications. Cambridge, UK: Cambridge University Press, 2001.

[8] M. Wada, J. Horikomi, and F. Mizutani, "Development of solid-state weather radar," in Proceedings of IEEE Radar Conference, Pasadena, CA, USA, May 2009, 4 pages.

[9] E. Kudeki and R. F. Woodman, "A poststatistics steering technique for MST radar applications," Radio Science, vol. 25, no. 4, pp. 591-594, Jul./Aug. 1990.

[10] R. D. Palmer, R. F. Woodman, S. Fukao, T. Tsuda, and S. Kato, "Threeantenna poststatistic steering using the MU radar," Radio Science, vol. 25, no. 6, pp. 1105-1110, Nov./Dec. 1990.

[11] E. Kudeki and F. Sürücü, "Radar interferometric imaging of field-aligned plasma irregularities in the equatorial electrojet," Geophysical Research Letters, vol. 18, no. 1, pp. 41-44, Jan. 1991.

[12] J. Capon, "High-resolution frequency-wavenumber spectrum analysis," Proceedings of the IEEE, vol. 57, no. 8, pp. 1408-1418, Aug. 1969.

[13] R. D. Palmer, S. Gopalam, T. Y. Yu, and S. Fukao, "Coherent radar imaging using Capon's method," Radio Science, vol. 33, no. 6, pp. 15851598, Nov./Dec. 1998.

[14] R. D. Palmer, T. Y. Yu, and P. B. Chilson, "Range imaging using frequency diversity," Radio Science, vol. 34, no. 6, pp. 1485-1496, Nov./Dec. 1999

[15] H. Luce, M. Yamamoto, S. Fukao, D. Helal, and M. Crochet, "A frequency domain radar interferometric imaging (FII) technique based on high-resolution methods," Journal of Atmospheric and Solar-Terrestrial Physics, vol. 63, no. 2-3, pp. 221-234, Jan. 2001.

[16] L. Cheong, M. W. Hoffman, and R. D. Palmer, "Efficient atmospheric simulation for high-resolution radar imaging applications," Journal of Atmospheric and Oceanic Technology, vol. 21, no. 2, pp. 374-378, Feb. 2004.

[17] B. L. Cheong, M. W. Hoffman, R. D. Palmer, S. J. Frasier, and F. J. López-Dekker, "Pulse pair beamforming and the effects of reflectivity field variations on imaging radars," Radio Science, vol. 39, no. 3 , 13 pages, Jun. 2004.
[18] L. Yang, M. R. McKay, R. Couillet, "High-dimensional MVDR beamforming: Optimized solutions based on spiked random matrix models," IEEE Transactions on Signal Processing, vol. 66, no. 7, pp. 1933-1947, Apr. 2018.

[19] E. Yoshikawa, T. Ushio, Z. Kawasaki, S. Yoshida, T. Morimoto, F. Mizutani, and M. Wada, "MMSE beam forming on fast-scanning phased array weather radar," IEEE Transactions on Geoscience and Remote Sensing, vol. 51, no. 5, pp. 3077-3088, May 2013.

[20] D. Kitahara, M. Nakahara, A. Hirabayashi, E. Yoshikawa, H. Kikuchi, and T. Ushio, "Nonlinear beamforming via convex optimization for phased array weather radar," in Proceedings of Asia-Pacific Signal and Information Processing Association Annual Summit and Conference (APSIPA ASC), Honolulu, HI, USA, Nov. 2018, pp. 1831-1835.

[21] D. Gabay and B. Mercier, "A dual algorithm for the solution of nonlinear variational problems via finite elements approximations," Computers \& Mathematics with Applications, vol. 2, no. 1, pp. 17-40, 1976.

[22] R. Glowinski, "On alternating direction methods of multipliers: A historical perspective," in Modeling, Simulation and Optimization for Science and Technology, W. Fitzgibbon, Y. A. Kuznetsov, P. Neittaanmäki, and O. Pironneau, Eds. Dordrecht, Netherlands: Springer Netherlands, 2014, pp. $59-82$.

[23] J. Eckstein and W. Yao, "Understanding the convergence of the alternating direction method of multipliers: Theoretical and computational perspectives," Pacific Journal of Optimization, vol. 11, no. 4, pp. 619644, Oct. 2015.

[24] L. Condat, D. Kitahara, A. Contreras, and A. Hirabayashi, "Proximal splitting algorithms: A tour of recent advances, with new twists," preprint arXiv:1912.00137, 46 pages, 2019.

[25] H. Kuroda, D. Kitahara, and A. Hirabayashi, "A convex penalty for block-sparse signals with unknown structures," in Proceedings of IEEE International Conference on Acoustics, Speech and Signal Processing (ICASSP), Toronto, Canada, Jun. 2021, 5 pages, to appear.

[26] F. J. Yanovsky, H. W. J. Russchenberg, and C. M. H. Unal, "Retrieval of information about turbulence in rain by using Doppler-polarimetric radar," IEEE Transactions on Microwave Theory and Techniques, vol. 53, no. 2, pp. 444-450, Feb. 2005.

[27] S. Fukao and K. Hamazu, Radar for Meteorological and Atmospheric Observations. Tokyo, Japan: Springer Japan, 2014.

[28] L. Jacob, G. Obozinski, and J. P. Vert, "Group lasso with overlap and graph lasso," in Proceedings of the 26th Annual International Conference on Machine Learning (ICML), Montreal, Canada, 2009, pp. 433440.

[29] G. Obozinski, L. Jacob, and J. P. Vert, "Group lasso with overlaps: the latent group lasso approach," preprint arXiv:1110.0413, 60 pages, 2011.

[30] F. Bach, R. Jenatton, J. Mairal, and G. Obozinski, "Optimization with sparsity-inducing penalties," Foundations and Trends in Machine Learning, vol. 4, no. 1, pp. 1-106, Jan. 2012.

[31] S. Villa, L. Rosasco, S. Mosci, and A. Verri, "Proximal methods for the latent group lasso penalty," Computational Optimization and Applications, vol. 58, pp. 381-407, 2014.

[32] W. W. Hager, "Updating the inverse of a matrix," SIAM Review, vol. 31, no. 2, pp. 221-239, Jun. 1989.

[33] G. H. Golub, and C. F. V. Loan, Matrix Computations, 4th ed. Baltimore, MD, USA: The Johns Hopkins University Press, 2013.

[34] E. Yoshikawa, N. Takizawa, H. Kikuchi, T. Mega, and T. Ushio, "An estimator for weather radar Doppler power spectrum via minimum mean square error," IEEE Transactions on Geoscience and Remote Sensing (Early Access), 16 pares, Jan. 2021.

[35] D. Middleton, An Introduction to Statistical Communication Theory. New York, NY, USA: McGraw-Hill, 1960.

[36] A. V. Oppenheim and R. W. Schafer, Discrete-Time Signal Processing, 3rd ed. Upper Saddle River, NJ, USA: Prentice Hall, 2009.

[37] N. Parikh and S. Boyd, "Proximal algorithms," Foundations and Trends in Machine Learning, vol. 1, no. 3, pp. 127-239, Jan. 2014. 


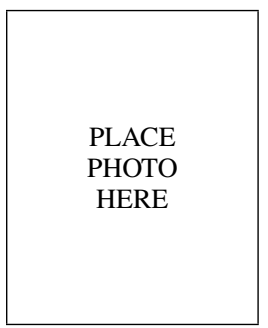

Daichi Kitahara

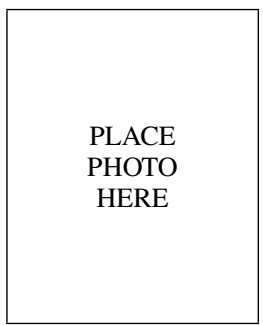

Tomoo Ushio

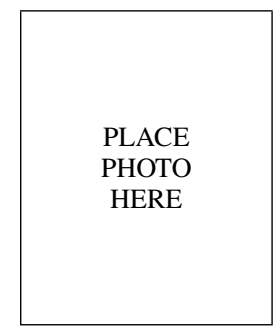

Hiroki Kuroda

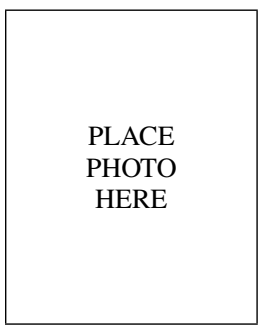

Akira Hirabayashi

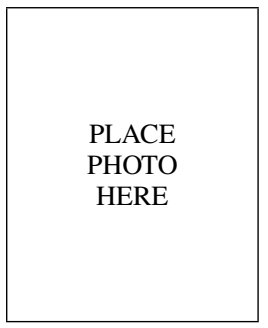

Eiichi Yoshikawa

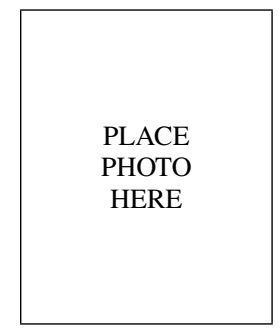

Hiroshi Kikuchi 